\title{
Nonlinear Dynamics of Vertical-Cavity Surface-Emitting Lasers
}

\author{
Krassimir Panajotov, ${ }^{1,2}$ Marc Sciamanna, ${ }^{3}$ Ignace Gatare, ${ }^{1}$ \\ Mikel Arteaga, ${ }^{4}$ and Hugo Thienpont ${ }^{1}$ \\ ${ }^{1}$ Department of Applied Physics and Photonics, Vrije Universiteit Brussel, Pleinlaan 2, 1050 Brussels, Belgium \\ ${ }^{2}$ Laboratory of Optics and Spectroscopy, Institute of Solid State Physics, 72 Tzarigradsko Chaussee Boulevard, 1784 Sofia, Bulgaria \\ ${ }^{3}$ Supélec, Optics \& Electronics (OPTEL), LMOPS EA-4423, 2 rue Edouard Belin, F-57070 Metz, France \\ ${ }^{4}$ Department of Electrical and Electronic Engineering, Public University of Navarra, 31006 Pamplona, Spain \\ Correspondence should be addressed to Krassimir Panajotov, kpanajot@b-phot.org
}

Received 6 June 2011; Accepted 28 July 2011

Academic Editor: Rainer Michalzik

Copyright ( $\odot 2011$ Krassimir Panajotov et al. This is an open access article distributed under the Creative Commons Attribution License, which permits unrestricted use, distribution, and reproduction in any medium, provided the original work is properly cited.

\begin{abstract}
Nonlinear dynamics of Vertical-Cavity Surface-Emitting Lasers (VCSELs) induced by optical injection, optical feedback, current modulation and mutual coupling is reviewed. Due to the surface emission and cylindrical symmetry VCSELs lack strong polarization anisotropy and may undergo polarization switching. Furthermore, VCSELs may emit light in multiple transverse modes. These VCSEL properties provide new features to the rich nonlinear dynamics induced by an external perturbation. We demonstrate for the case of orthogonal optical injection that new Hopf bifurcation on a two-polarization-mode solution delimits the injection locking region and that polarization switching and injection locking of first-order transverse mode lead to a new resonance tongue for large positive detunings. Similarly, the underlying polarization mode competition leads to chaoticlike behavior in case of gain switching and the presence of two transverse modes additionally reduces the possibility of regular dynamics. The bistable property of VCSEL makes it possible to investigate very fundamental problems of bistable systems with time-delay, such as the coherence resonance phenomenon. We also demonstrate that the synchronization quality between unidirectionally coupled VCSELs can be significantly enhanced when the feedback-induced chaos in the master laser involves both orthogonal LP fundamental transverse modes.
\end{abstract}

\section{Introduction}

The change of the semiconductor laser cavity geometry from facet to surface-emitting gave birth to the verticalcavity surface-emitting lasers (VCSELs) [1] with significant advantages, such as single longitudinal mode emission, low cost, circular output beam, and easy fabrication in twodimensional arrays. As a result, VCSELs are nowadays substituting the traditional semiconductor edge emitting lasers (EELs) in many applications, such as fiber to the home links, computer networks, optical interconnects, and optical sensing. Until recently, most of the commercially available VCSELs were emitting in the near infrared, around $850 \mathrm{~nm}$ or $970 \mathrm{~nm}$, based on GaAs active region and GaAs/AlGaAs DBR mirrors but now they become available also at telecommunication wavelengths 1.3 or $1.55 \mu \mathrm{m}$ and at visible or even UV wavelengths. Emission in multiple transverse modes is usually found in VCSELs [2] as a result of spatial hole burning effect $[3,4]$. Furthermore, due to the surface emission and cylindrical symmetry VCSELs grown on (001) substrate lack strong polarization anisotropy and may undergo polarization switching (PS) [2, 5]. Different physical mechanisms can lead to PS, such as microscopic spinflip processes in presence of birefringence and linewidth enhancement factor [6, 7], thermal lensing [8], spatial hole burning [9], or the relative modification of the net modal gain and losses with the injection current $[5,10]$. The lack of polarization anisotropies and the multitransverse mode behavior of VCSELs provide new features to the rich nonlinear dynamics induced by optical injection (OI), gain switching (GS), and optical feedback (OF).

Locking of the frequency of the injected (slave) semiconductor laser to the one of the injecting (master) laser has long been known $[11,12]$ and is of great interest from an application point of view. It can be used for reduction of the laser linewidth [12] and the mode partition noise 
[13] or for an enhancement of the modulation bandwidth [14] and for synchronizing an array of lasers onto a unique master [15]. Furthermore, OI dynamics is also of interest from a fundamental point of view and rich dynamics as period doubling route to chaos [16-18], resonance tongues [19], and excitability [20] have been reported. Orthogonal OI in VCSELs, that is, the linear polarization of the injected light is orthogonal to the one of the VCSEL, was first reported by Pan et al. [21] in 1993. It was shown that increasing the injection strength, the VCSEL switches its polarization to that of the injected light through a region of bistability and may exhibit an injection locking (IL) depending on the frequency detuning between the two lasers [22]. Hong et al. [23] have shown that the otherwise depressed LP mode may be excited by orthogonal OI and regions of chaotic competitions in the two linearly polarized (LP) modes of the VCSEL have been shown experimentally both for positive and negative detunings. The case of parallel OI has been investigated by Li et al. [24]. Transverse mode dynamics of a VCSEL with OI have been analyzed numerically [25] and experimentally $[26,27]$ for different configurations of the polarization of the injected light with respect to the light polarization of the freerunning VCSEL.

Gain switching of semiconductor lasers has received a lot of attention, considering not only its potential to generate ultrafast sharp pulses but also because it can lead to complex dynamics such as period doubling and possibly chaotic pulsating [28-31]. Just a few reports of chaotic behavior can be found in the literature $[30,31]$ since only lasers with relatively small gain saturation and spontaneous emission noise parameters might undergo period doubling route to chaos under current modulation [32]. Studies of nonlinear dynamics in directly modulated VCSELs remain scarce [3335]. Nonlinear dynamics have been theoretically analyzed for linearly polarized (LP) single transverse mode [33, 34] and multimode VCSELs $[34,35]$. Chaotic behaviour appears in the multimode regime due to transverse mode competition $[34,35]$. Only recently, theoretical and experimental studies have been undertaken with special attention on the role of light polarization [36-39].

Time-delayed optical feedback (OF) perturbs the steady state laser emission and leads to complex dynamics [40]. It can lead to quite irregular (chaotic) dynamics [41] and as much as 3 orders of magnitude increase of the optical linewidth [42]. The same sequence of dynamical regimes that has been observed in EEL [42] is also reported in VCSELs [43]. With increasing the feedback strength these are linewidth narrowing or broadening; external-cavity mode (ECM) hopping; stable emission; sustained relaxation oscillations; chaotic regimes (low-frequency fluctuations, coherence collapse). Low-frequency fluctuation (LFF) dynamics [41] consists of sudden dropouts in the emitted optical power followed by gradual recoveries. LFF in VCSELs was studied theoretically in $[44,45]$ and found experimentally in $[46,47]$. The effect of optical feedback on the transverse mode selection in VCSELs has also been investigated by several authors, for example, in $[48,49]$.

Synchronization of chaotic systems has been a subject of both theoretical and experimental studies [50]. In particular, the synchronization between two chaotic semiconductor lasers has attracted considerable attention owing to its application in secure chaos-based optical communications [51]. Recent theoretical works have focused on the synchronization properties in polarization-dependent optical injection schemes [52] or using multitransverse mode but not single LP mode VCSELs [53]. Chaos synchronization has also been recently shown experimentally [54]. The growing interest in implementing chaos communication using VCSELs motivates further investigations to better understand the influence of polarization dynamics on the synchronization properties.

In this paper we summarize recent results on nonlinear dynamics of VCSELs for the case of optical injection, gain switching, optical feedback, and unidirectional chaos synchronization. The paper is organized as follows: in Section 2, we demonstrate that, for the case of orthogonal OI, a new Hopf bifurcation on a two-polarizationmode solution delimits the injection locking region and that polarization switching and injection locking of firstorder transverse mode lead to a new resonance tongue for large positive detunings. In Section 3 we show that the underlying polarization mode competition leads to chaotic-like behavior in case of gain switching and the presence of two transverse modes additionally reduces the possibility of regular dynamics as shown. In Section 4 we discuss optical feedback-induced polarization dynamics that allows investigating very fundamental problems of bistable systems with time delay, such as the coherence resonance phenomenon. In Section 5 we demonstrate that the synchronization quality between unidirectionally coupled VCSELs can be significantly enhanced when the feedback-induced chaos in the master laser involves both orthogonal LP fundamental transverse modes. Finally, in Section 6, a brief summary of the results is presented.

\section{Nonlinear Polarization and Transverse Mode Dynamics in VCSEL with Orthogonal Optical Injection}

The experiments on orthogonal OI have been performed with oxide-confined GaAs quantum well VCSEL emitting at $845 \mathrm{~nm}$ as a slave laser (SL). The solitary VCSEL is polarization bistable in the injection current region $2.25 \mathrm{~mA}-$ $4.60 \mathrm{~mA}$, displaying type II PS (from the low to the high frequency LP mode [10]). It is biased below the bistable region, at $\mathrm{I}=2.105 \mathrm{~mA}$, and emits fundamental transverse mode with horizontal polarization $(x$-LP) and output power of $P_{\text {out }}=1.28 \mathrm{~mW}$. An external cavity tunable laser is used as a master laser (ML) and is set to emit vertically polarized light $(y$-LP).

In Figure 1 we show a typical route to PS through injection locking by presenting high resolution Fabry-Perot spectra for different injected powers and fixed negative detuning of $-4 \mathrm{GHz}$ [22]. For very low injected power, the VCSEL keeps emitting in $x$-LP but is pulled toward the ML frequency proportionally to the strength of the injection. An increase of the injected power leads to undamping of relaxation oscillations-small side peaks appear on each side of the SL 


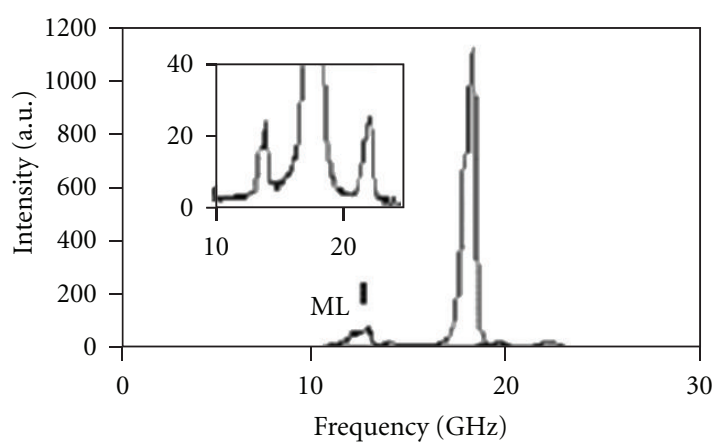

(a)

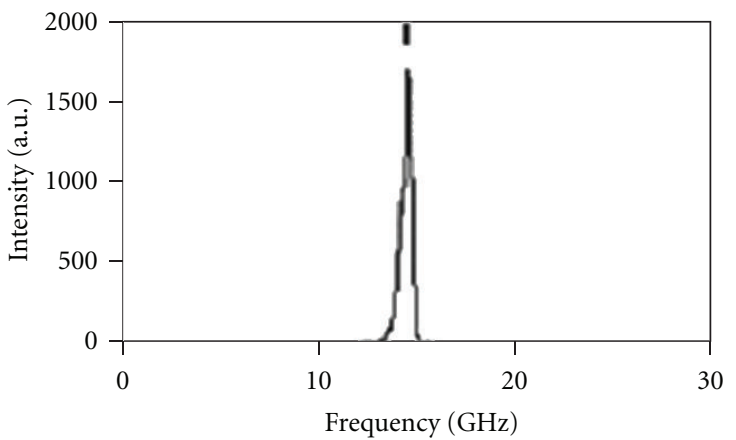

(b)

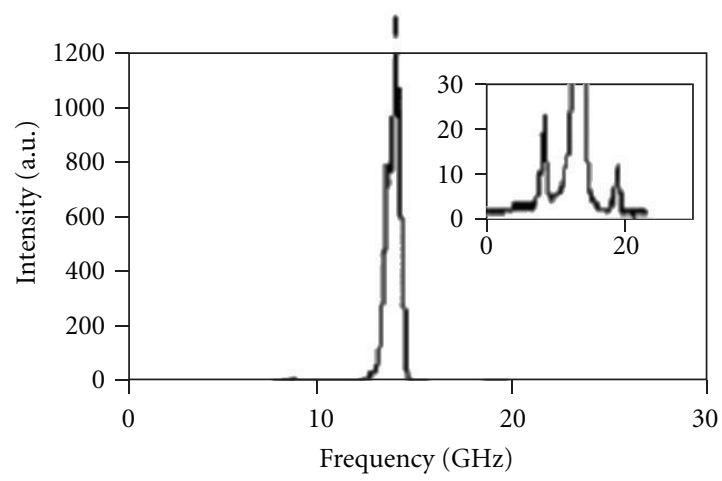

(c)

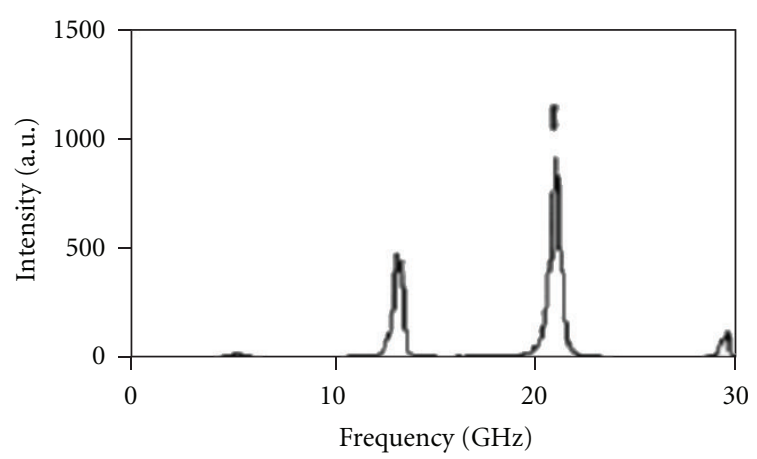

(d)

FIgURE 1: Polarization-resolved optical spectra of the VCSEL subject to optical injection at a frequency detuning of $-4 \mathrm{GHz}$. (a) $P_{\text {inj }}=40 \mu \mathrm{W}$. (b) $76 \mu \mathrm{W}$. (c) $103 \mu \mathrm{W}$. (d) $210 \mu \mathrm{W}$. The vertical line shows the ML frequency. The insets in (a) and (c) show the same spectra magnified. After [22]. (Reprinted by permission from IEEE J. Quantum Electronics.)

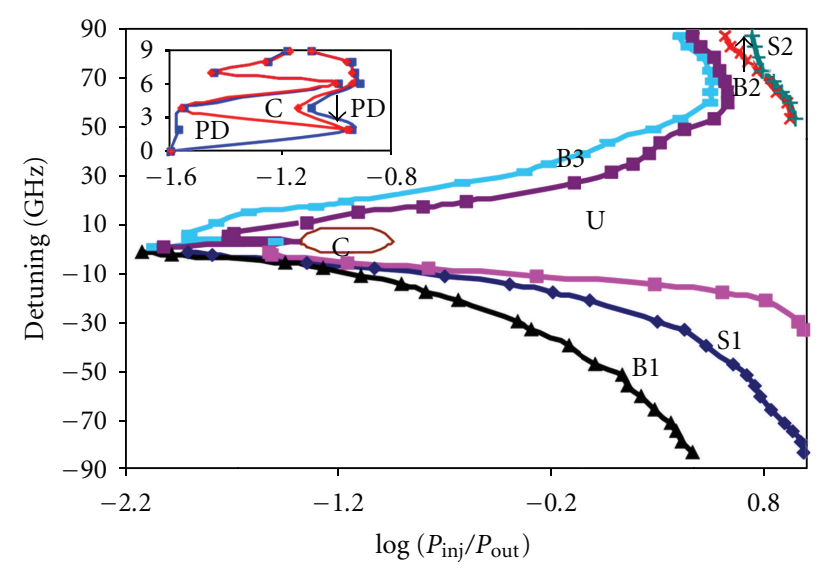

FIGURE 2: Experimental mapping of bifurcations to qualitatively different polarization dynamics in a VCSEL subject to orthogonal optical injection. Labels and symbols are explained in the text. After [55]. (Reprinted by permission from Appl. Phys. Lett.)

peak (Figure 1(a)). For still larger injection strength, PS with injection frequency locking of the fundamental transverse mode is achieved (Figure $1(\mathrm{~b})$ ). The locking region is excited by excitation of a limit cycle dynamics (Figure 1(c)). Harmonics of the limit cycle are resolved for higher injection power, and further the slave laser (SL) frequency is progressively pushed from the ML frequency (Figure 1(d)).

Figure 2 presents the boundaries of qualitatively different dynamics experimentally mapped in the plane of frequency detuning (master minus the slave laser frequency)_injection power (normalized to the solitary VCSEL power) $[22,55]$. The polarization switch off and switch on points of the $x$-LP mode for increasing (decreasing) the injected power are represented by the dark blue and violet (light blue and black) lines. In the regions S1 and S2, the frequency of VCSEL emission is locked to the master laser. However, in the case of S2, it is the first order transverse mode rather than the fundamental transverse mode (as is the case of the S1 region) that locks to the master laser, the fundamental transverse mode being then suppressed when crossing the dark green line. The unlocking of the first order transverse mode happens at smaller values of $P_{\text {inj }}$, describing bistable region $\mathrm{B} 2$ between the fundamental and the first order transverse mode both with the same polarization (B2 is delimited by the dark green and red lines). We observe two polarization bistable regions in a regime of fundamental mode emission, which correspond to two different ways of PS. The first one is with frequency locking (B1), as presented in Figure 1, and is confined between the dark blue and the black lines. The second polarization bistable region (B3) is confined between the light blue and the violet lines where the PS happens without frequency locking. The two bistable regions are connected at a detuning of $2 \mathrm{GHz}$, which coincides with the birefringence frequency splitting between the two VCSEL LP modes. The bistable region B3 is strongly influenced by the locking of the first order LP mode (S2), its borderline turning backwards at a detuning of $50 \mathrm{GHz}$. The mapping of dynamical states shows that rich nonlinear dynamics including period-doubling route to 
chaos and reverse period-doubling from the chaotic zone are found for detunings in the range $2-10 \mathrm{GHz}$ (see the inset of Figure 2 and also [22]).

In Figure 3, the mapping of the VCSEL subject to optical injection is extended towards large positive detuning up to $180 \mathrm{GHz}$ [56]. When the injection strength is increased, different PS scenarios are resolved depending on the frequency detuning. A switching from $x$-LP to $y$-LP fundamental transverse mode is observed for the whole frequency detuning range-denoted by black triangles in Figure 3. This boundary exhibits two minima for the switching power: the first located at a detuning of $2 \mathrm{GHz}$ and the second one at a detuning of $150 \mathrm{GHz}$ with injection powers of $7.1 \mu \mathrm{W}$ and $623.9 \mu \mathrm{W}$, respectively. Injection locking of the first-order transverse mode together with suppression of the fundamental mode is observed for frequency detuning range from 60 to $120 \mathrm{GHz}$-denoted by black diamonds in Figure 3.

It is possible to reproduce theoretically the experimental results on the base of a set of rate equations that accounts for the polarization properties of VCSELs, namely, the spin-flip model (SFM) [6]. The SFM equations [6, 7], extended to the case of optical injection are given in $[7,57-59]$ for a singletransverse-mode VCSEL and in [56] for a multitransversemode VCSEL. For completeness, we list the SFM equations for the case of OI in a single-transverse mode VCSEL, namely,

$$
\begin{aligned}
\frac{d E_{x}}{d t}= & -\left(\kappa+\gamma_{a}\right) E_{x}-i\left(\kappa \alpha+\gamma_{p}+\Delta \omega\right) E_{x} \\
& +\kappa(1+i \alpha)\left(N E_{x}+i n E_{y}\right), \\
\frac{d E_{y}}{d t}= & -\left(\kappa-\gamma_{a}\right) E_{y}-i\left(\kappa \alpha-\gamma_{p}-\Delta \omega\right) E_{y} \\
& +\kappa(1+i \alpha)\left(N E_{y}-i n E_{x}\right)+\kappa_{\mathrm{inj}} E_{\mathrm{inj}}, \\
\frac{d N}{d t}= & -\gamma\left[N\left(1+\left|E_{x}\right|^{2}+\left|E_{y}\right|^{2}\right)\right. \\
& \left.\quad-\mu+i n\left(E_{y} E_{x}^{*}-E_{y} E_{x}^{*}\right)\right], \\
\frac{d n}{d t}= & -\gamma_{s} n-\gamma\left[n\left(\left|E_{x}\right|^{2}+\left|E_{y}\right|^{2}\right)+i N\left(E_{y} E_{x}^{*}-E_{y} E_{x}^{*}\right)\right] .
\end{aligned}
$$

In addition to the coupling of the polarizations states through the carrier density (3), the SFM accounts for a coupling due to the finite spin-flip rate $\gamma_{s}$ of the carriers [5], which, together with the linewidth enhancement factor $\alpha$ and the inherent small VCSEL birefringence $\gamma_{p}=\left(\omega_{y}-\right.$ $\left.\omega_{x}\right) / 2$ and dichroism $\gamma_{a}$ leads to PS in a solitary laser [7]. The variable $N$ is related to the total inversion between conduction and valence bands, while $n$ accounts for the difference in the carrier numbers with opposite spins. The rest of the parameters are $\mu$ : the normalized injection current; $\kappa$ : the photon decay rate; $\gamma$ : the carrier decay rate. OI of $y$-LP light is accounted for by $\kappa_{\text {inj }}$ : the coupling coefficient, $E_{\text {inj }}$ : the injected field amplitude, and $\Delta \omega=\omega_{\text {inj }}-(\omega x+\omega y) / 2-$ the

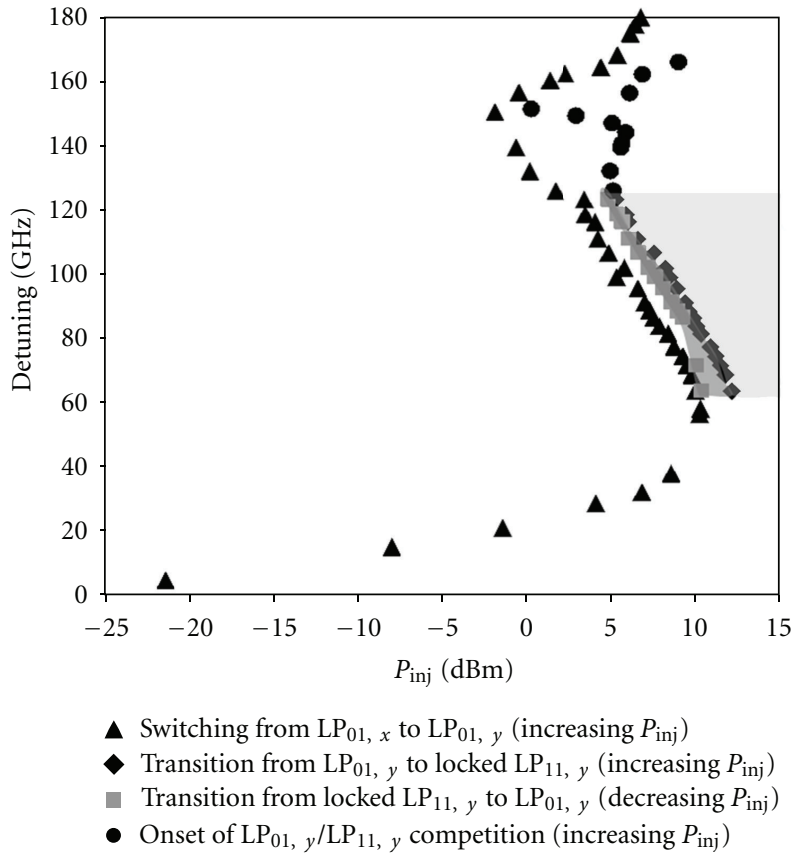

FIGURE 3: Experimental mapping of polarization switching and transverse modes competition extended towards large positive detunings. After [56]. (Reprinted, by permission, from IEEE J. Quantum Electronics.)

frequency detuning between the master and the mean of the VCSEL LP mode frequencies.

Figure 4 shows a typical bifurcation mapping for a singletransverse-mode VCSEL with parameters $\mu=1.5, \kappa=$ $300 \mathrm{~ns}^{-1}, \gamma=1 \mathrm{~ns}^{-1}, \gamma_{p}=30 \mathrm{~ns}^{-1}, \gamma_{a}=0.5 \mathrm{~ns}^{-1}$, and $\gamma_{s}=100 \mathrm{~ns}^{-1}$. Qualitative changes in the VCSEL dynamics are detected and followed using the continuation package AUTO 97. Different bifurcation curves are plotted: a saddlenode $(\mathrm{SN})$, two Hopf $\left(\mathrm{H}_{1}\right.$ and $\left.\mathrm{H}_{2}\right)$, and a torus (TR). The supercritical and subcritical parts of each bifurcation curve are represented in black and gray, respectively. When increasing the injection strength, the VCSEL switches its polarization to that of the injected field; these "PS off" ( $x$-LP mode off) points are shown with circles. The PS curves interplay with the bifurcation curves. $\mathrm{SN}$ and $\mathrm{H}_{1}$ are bifurcations on a stationary injection-locked state and have also been reported in the case of optically injected edge-emitting laser. In the conventional case of EEL, the locking region is then delimited by the codimension twopoint $\mathrm{G}_{1}$ where $\mathrm{SN}$ and $\mathrm{H}_{1}$ intersect [60]. In our VCSEL system, the locking region is delimited not only by $\mathrm{SN}, \mathrm{H}_{1}$ but also by a new bifurcation $\mathrm{H}_{2}$ (see Figure 4(b)). The maximum detuning leading to injection locking therefore stays well below the codimension-two saddle-node-Hopf point $\mathrm{G}_{1}$. Apart from its effect on the locking, $\mathrm{H}_{2}$ also affects the PS mechanism. The smallest injection strength needed to achieve PS is located on $\mathrm{H}_{2}$ and corresponds to a dramatic change in the PS curve $\left(m_{1}\right.$, see the dot vertical arrow in Figure 4(c)). The regions of more complicated, possibly chaotic dynamics bounded by $\mathrm{PD}_{1}$ and $\mathrm{PD}_{2}$, also affect the switching mechanism leading to a second local 


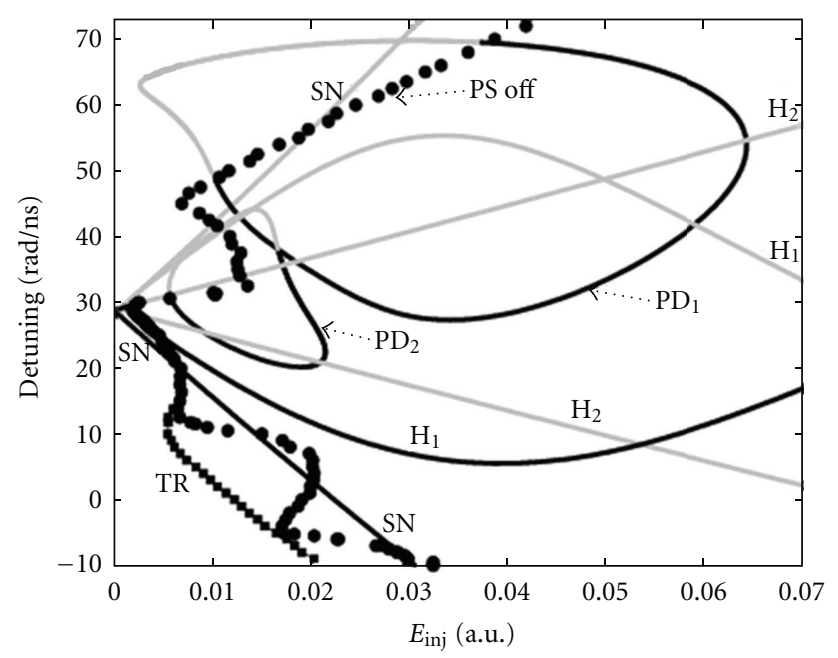

(a)

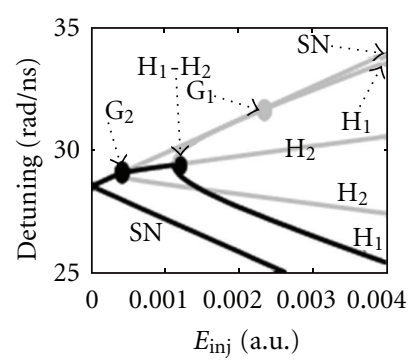

(b)

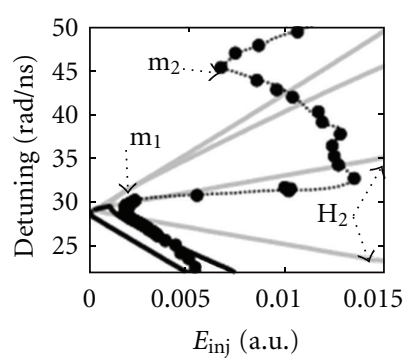

(c)
FIGURE 4: Bifurcation diagram in the plane injection strengthfrequency detuning calculated by the SFM model for $\kappa=300 \mathrm{~ns}^{-1}$, $\gamma_{p}=30 \mathrm{~ns}^{-1}, \gamma_{a}=0.5 \mathrm{~ns}^{-1}, \gamma=1 \mathrm{~ns}^{-1}, \gamma_{s}=100 \mathrm{~ns}^{-1}$ and $\mu=1.5$. Close view of the codimension-two bifurcation mechanisms (b) and of the wobbling shape in the switching curve with local minima indicated by thick dotted arrows (c). (TR; "PS off") and (TR) curves have been removed, respectively, in panels (b) and (c) for clarity. Black (gray) denotes supercritical (subcritical) bifurcation. After [57]. (Reprinted by permission from Phys. Rev. A.)

minimum denoted by $m_{2}$ in Figure 4(c). As a result, the PS curve exhibits a wobbling shape with local minima of the injected power required for switching. The observed shape agrees qualitatively with the experimental results presented in Figure 2. The torus bifurcation TR corresponds to the excitation of two polarization mode dynamics in the route to PS and injection locking - a limit cycle in the $x$-LP and a wave mixing in the $y$-LP-which is also in agreement with the experiment [57]. The SFM model further predicts a twomode injection-locked solution, that is, elliptically polarized injection-locked state for frequency detuning close to the VCSEL birefringence [58]. The bifurcation route to such solution has been studied in detail in [57].

\section{Nonlinear Polarization and Transverse Mode Dynamics in Gain-Switched VCSEL}

Polarization dynamics of gain-switched VCSELs was first investigated in [36] based on the spin-flip model for a single-

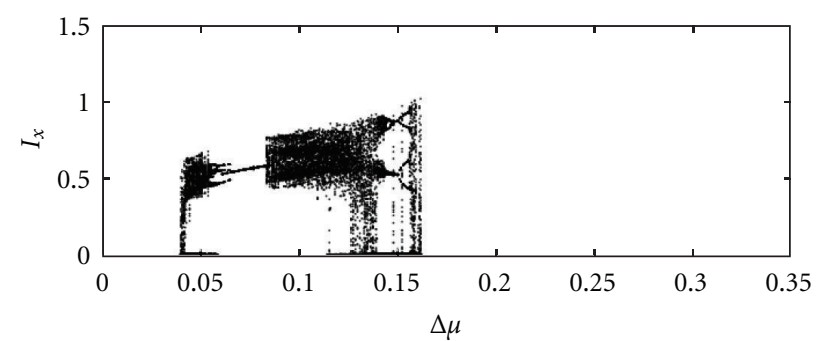

(a)

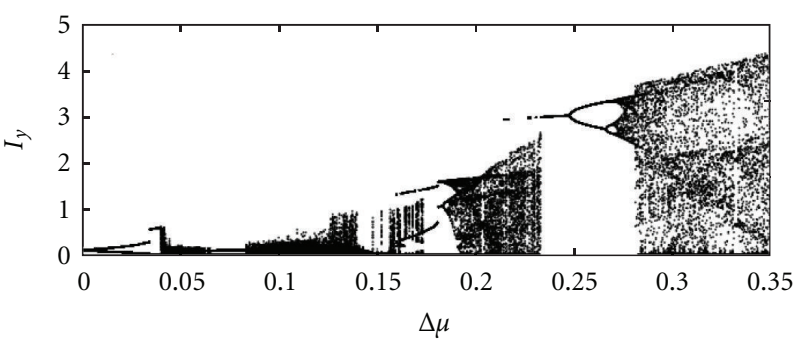

(b)

FIGURE 5: Bifurcation diagrams of the power of $x$-LP mode (a) and the $y$-LP mode (b) with the modulation amplitude $\Delta \mu$ obtained by the SFM model for a single-mode VCSEL. The bias current is $\mu_{d c}=1.1$ and the modulation frequency is $\nu_{M}=1 \mathrm{GHz}$. The VCSEL parameters are specified in the text. After [36]. (Reprinted by permission from Phys. Rev. E.)

transverse mode VCSEL given by (1)-(4) but without the optical injection term $\left(\Delta \omega=0\right.$ and $E_{\text {inj }}=0$ in (2)) and with sinusoidally modulated injection current in (3):

$$
\mu=\mu_{d c}+\Delta \mu \sin \left(2 \pi v_{M} t\right),
$$

where $\mu_{d c}$ is the bias current and $\Delta \mu$ and $\nu_{M}$ are the amplitude and the frequency of modulation of the injection current, respectively.

Bifurcation diagrams of the polarization-resolved output power $I_{x}$ and $I_{y}$ are shown in Figure 5 as a function of amplitude of modulation $\Delta \mu$ for $\nu_{M}=1 \mathrm{GHz}$ and $\mu_{d c}=1$.1. The VCSEL parameters are $\alpha=3, \gamma_{a}=0.1 \mathrm{~ns}^{-1}, \gamma_{p}=1 \mathrm{~ns}^{-1}, \gamma_{s}=$ $50 \mathrm{~ns}^{-1}, \kappa=300 \mathrm{~ns}^{-1}, \gamma=1 \mathrm{~ns}^{-1}$. The modulation frequency $\nu_{M}$ is smaller than the relaxation oscillation frequency of the VCSEL $\nu_{\mathrm{RO}}=[2 \kappa \gamma(\mu-1)]^{1 / 2} / 2 \pi=1.23 \mathrm{GHz}$. As can be seen from Figure 5, the VCSEL initially lases in the $y$-LP mode, with increasing amplitude of sinusoidally modulated intensity at the modulation period with increasing $\Delta \mu$. For large enough $\Delta \mu$, such that the injection current goes from below to above the threshold current, gain switching occurs. In certain regions of $\Delta \mu$, the two LP modes coexist with chaotic or time-periodic dynamics and for still larger $\Delta \mu$ the VCSEL lases only in the $y$-LP mode with a period-doubling route to chaos.

The LP mode dynamics is detailed in Figure 6, which shows time traces of the polarization resolved intensities for specific values of $\Delta \mu$. At $\Delta \mu=0.04$, the depressed $x$-LP mode is also lasing, that is, the direct current modulation has excited the LP mode that was depressed in the VCSEL under dc operation. The two LP modes exhibit chaotic-like 


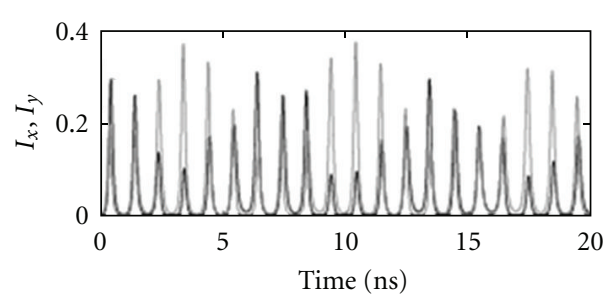

(a)

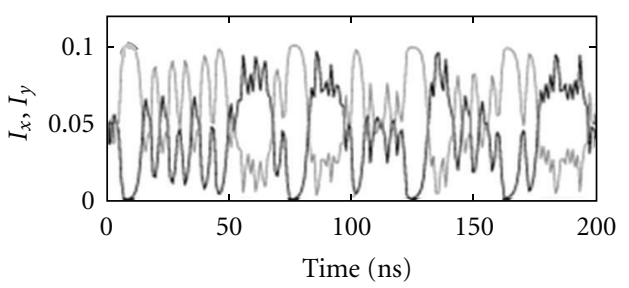

(b)

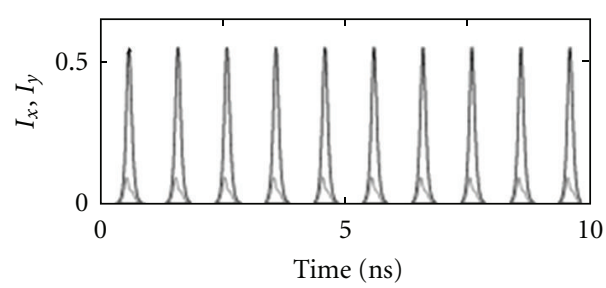

(c)

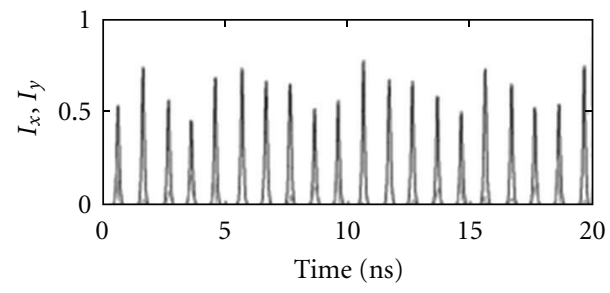

(d)

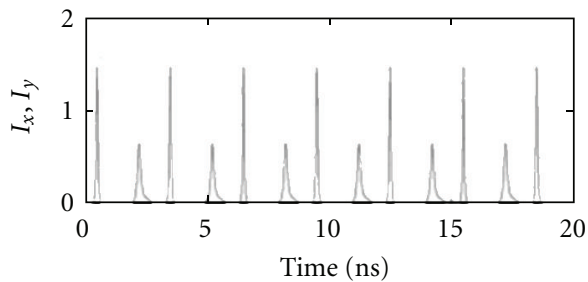

(e)

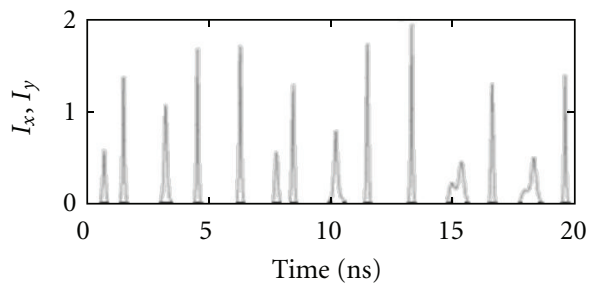

(f)

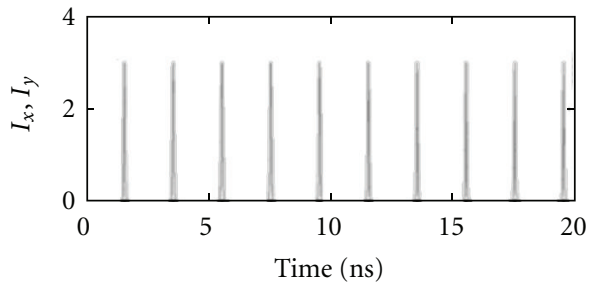

(g)

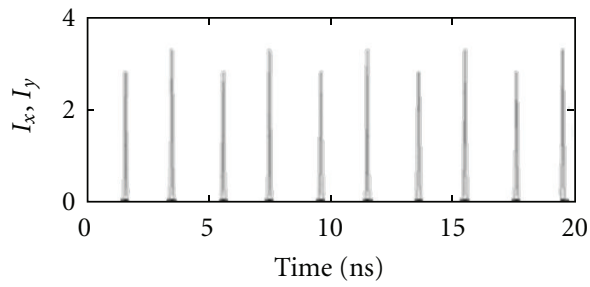

(h)

Figure 6: Time traces of $I_{x}$ (black) and $I_{y}$ (gray) for specific values of $\Delta \mu$ in Figure 7: (a) and (b) $\Delta \mu=0.04$, (c) $\Delta \mu=0.07$, (d) $\Delta \mu=0.1$, (e) $\Delta \mu=0.18$, (f) $\Delta \mu=0.2$, (g) $\Delta \mu=0.24$, (h) $\Delta \mu=0.26$. (b) is the same as (a) but averaged over 1 ns. After [36]. (Reprinted by permission from Phys. Rev. E.)

dynamics with a fast modulation of their intensities at the modulation frequency complemented by a slower envelope. Interestingly, the two LP modes emit in phase at the modulation frequency but the envelopes of their pulses are in partial antiphase: when one LP mode fires a large pulse, the other LP mode fires a small pulse. The partial antiphase dynamics is better seen in Figure 6(b), which presents the averaged over the modulation period output power in the two LP modes. For a slightly larger $\Delta \mu=0.07$, Figure 6(c), the two LP modes exhibit in-phase time-periodic pulsing at the modulation frequency. For larger $\Delta \mu$, the $y$-LP mode is only lasing. At $\Delta \mu=0.18$, Figure 6(e), the $y$-LP mode exhibits a period-3 time-periodic pulsing dynamics, which is followed by chaotic single mode dynamics at $\Delta \mu=0.2$, Figure 6(f). For increasing modulation depth, the dynamics is single period one, Figure $6(\mathrm{~g})$, followed by a period-doubling route to chaos-the period 2 is shown for $\Delta \mu=0.24$ in Figure 6(h).

These theoretical predictions have been experimentally confirmed in [37]. A GaAs quantum-well oxide-confined VCSEL emitting at $851 \mathrm{~nm}$ is used in the experiments biased close to the PS point and then driven by an RF-signal thorough a bias-T. The temporal traces of the total and polarized powers are shown in Figure 7 for a modulation frequency of $2.88 \mathrm{GHz}$ and current amplitude of $\Delta I=$ $0.89 I_{\text {th }}$. A regular stream of pulses at each two periods of modulation is found for the total power. Small shoulders appear, that are remnants of the period- 1 dynamics. When the amplitude of the modulation decreases, those shoulders become larger until similar heights are obtained for all the pulses in such a way that only one pulse appears each modulation cycle (period-1 dynamics). Pulses in individual polarizations also appear with that periodicity but their 


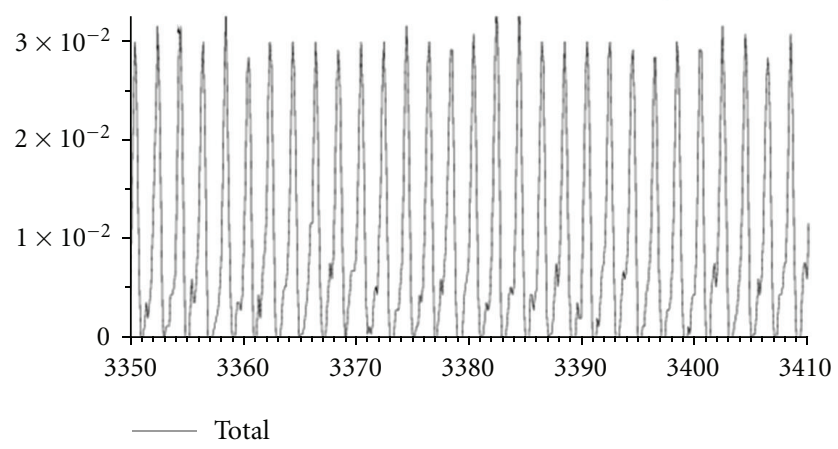

(a)

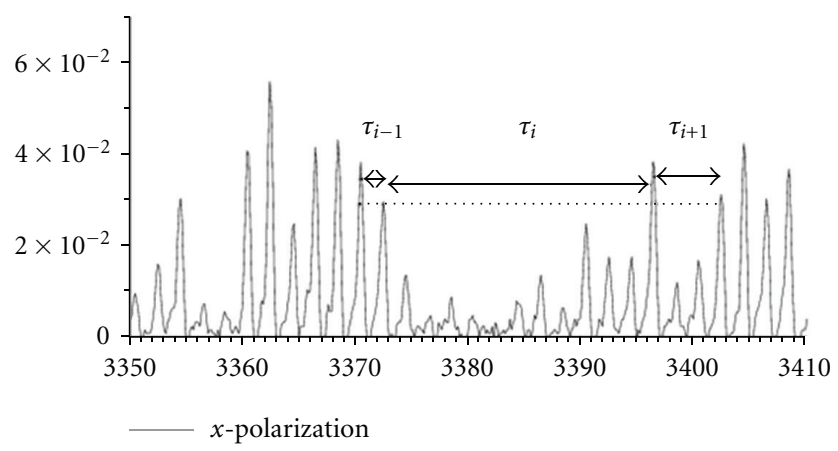

(b)

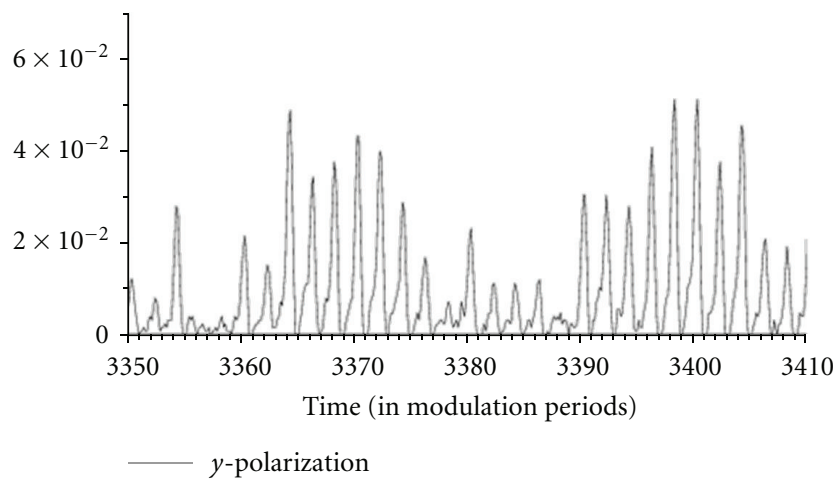

(c)

Figure 7: Experimental time traces of the intensities of the total (a), $x$-polarized (b), and $y$-polarized (c) powers. The current modulation is such that $\nu_{M}=2.88 \mathrm{GHz}, I=1.26 I_{\text {th }}$, and $\Delta I=0.89 I_{\text {th }}$. After [37]. (Reprinted by permission from IEEE J. Quantum Electronics.)

heights are very irregular with wide temporal regions in which pulses in one polarization are very small while in orthogonal polarization are very high-as deduced from the regularity of the total power. Another indication for the regularity of the pulse dynamics is the statistical distribution of the residence times $\tau_{x}\left(\tau_{y}\right)$ given by the time between consecutive crossings of the corresponding $P_{x}\left(P_{y}\right)$ output power, from below to above, of certain reference level chosen as half of the total power maximum (see Figure 7(b)). Both the experimental and theoretical probability density

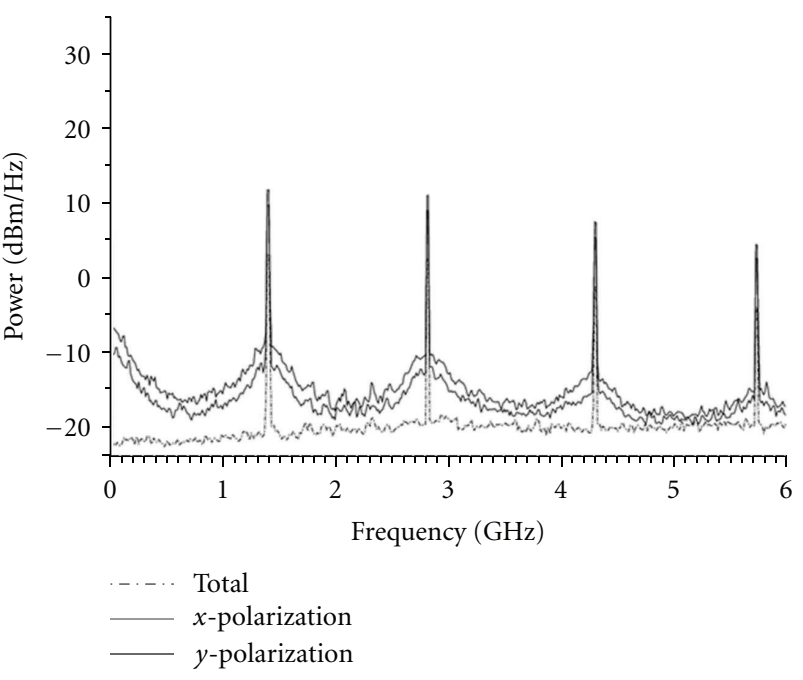

Figure 8: Experimental RF spectra of the total, $x$-polarized, and $y$ polarized power. The parameters of the modulation are the same as in Figure 7. After [37]. (Reprinted by permission from IEEE J. Quantum Electronics.)

distributions of the residence times for the individual polarizations present a multipeaked structure with a long exponential envelope [37]. This long exponential tail is a signature of the irregularity of the stream of pulses-in contrast with the absence of such a tail for the total power.

The irregular behavior of individual polarizations, in contrast to the regular one of the total power, is also manifested in the corresponding RF spectra shown in Figure 8. Large peaks appear half of the modulation frequency and its multiples. These peaks are an indication of the period-doubling dynamics observed in the time series in Figure 7. The large pedestals that appear around the peaks of the spectra of individual polarizations indicate that the polarization-resolved dynamics is quite irregular. The power levels at low frequencies are much larger for the individual polarizations than for the total power, as a result of the anticorrelation between the two polarizations.

Gain-switched multitransverse mode VCSEL has been considered in [38]. The calculated bifurcation diagrams for the total and polarization resolved powers of the fundamental and first-order transverse modes display periodic and chaotic dynamics as well as for the total intensity. The $\mathrm{LP}_{11}$ mode is now excited with a significant power for all the modulation amplitudes. Time-periodic dynamics is now restricted to low values of $\Delta \mu$, that is, the excitation of the higher order transverse mode causes a disappearance of the windows of time-periodic regular pulsating dynamics found in the single-mode case.

These theoretical predictions were experimentally confirmed by using a GaAs quantum well VCSEL with medium oxide aperture size, such that it can operate on two transverse-modes simultaneously but in one linear polarization only [39]. A sinusoidal voltage is used to modulate the current around a bias value of $5.3 \mathrm{~mA}$. Time traces obtained in the vertical polarization for a fixed amplitude 


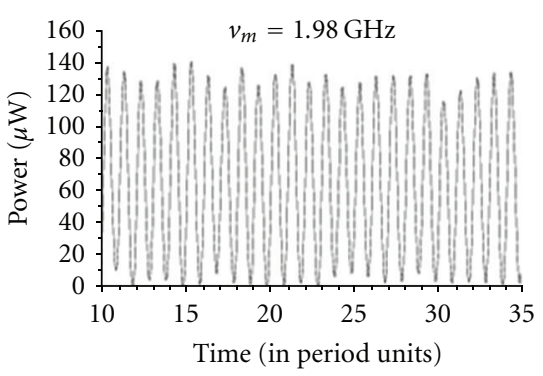

(a)

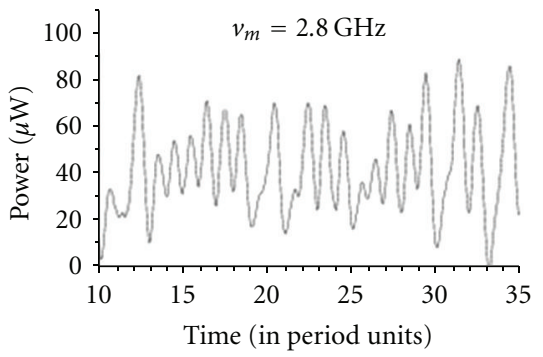

(d)

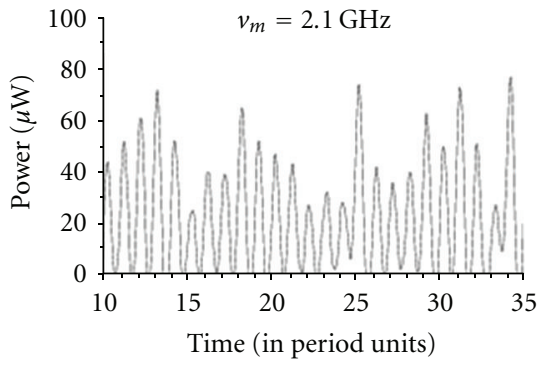

(b)

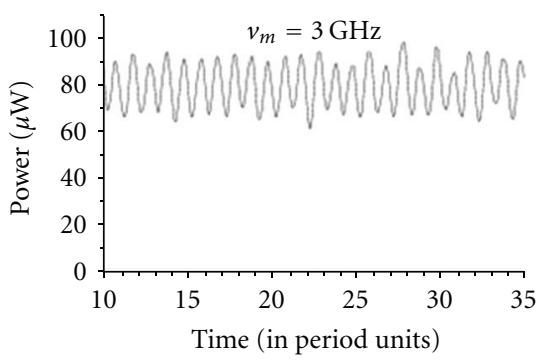

(e)

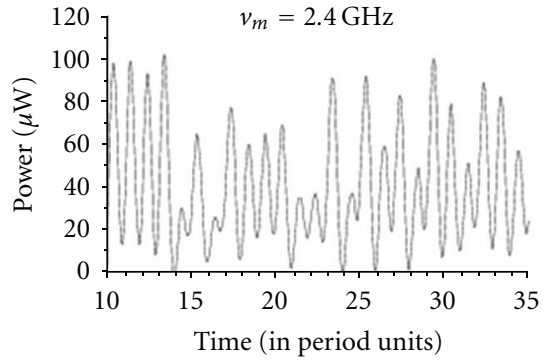

(c)

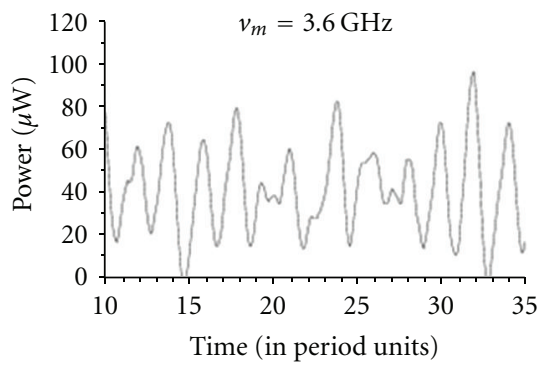

(f)

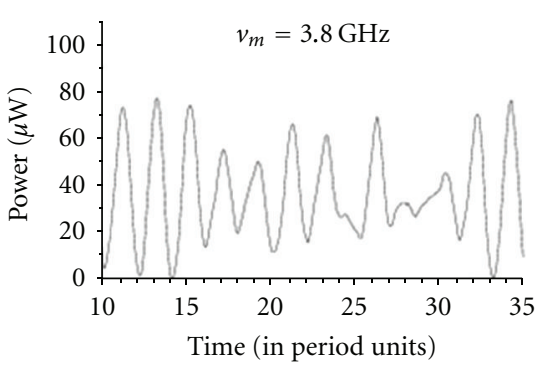

(g)

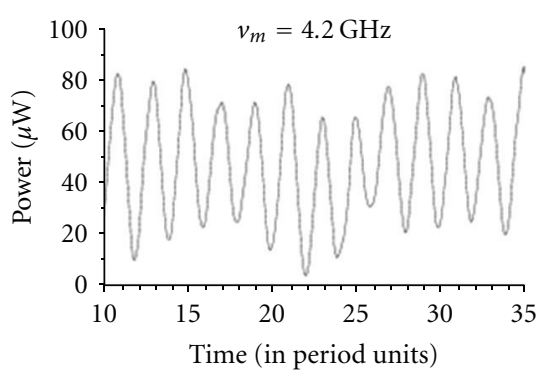

(h)

Figure 9: Time traces of the VCSEL output power for several values of the modulation frequency $\nu_{M}$, as denoted. The amplitude of the modulation is $20 \mathrm{dBm}$. After [39]. (Reprinted, by permission, from Appl. Phys. Lett.)

of $20 \mathrm{dBm}$ - large enough to achieve gain-switching—and several modulation frequencies are shown in Figure 9. Timeperiodic response of the system, with a period equal to the period of the modulation is obtained when $v_{M}=1.98 \mathrm{GHz}$ (Figure 9(a)). This situation is maintained in Figures 9(b), 9(c), and 9(d) but the pulse heights change in irregular way. 1T-periodic response with smaller modulation amplitudes again appears at $\nu_{M}=3 \mathrm{GHz}$ (Figure 9(e)) followed by irregular responses when increasing $\nu_{M}$ (Figures 9(f) and $9(\mathrm{~g}))$. The difference with respect to the irregular responses of Figures $9(\mathrm{~b})$ and $9(\mathrm{c})$ is that now some of the pulses appear at $2 \mathrm{~T}$. For $\nu_{M}=4.2 \mathrm{GHz}$ in Figure $9(\mathrm{~h})$, a more regular response but with a $2 \mathrm{~T}$ periodicity is observed. We have measured a $2.4 \mathrm{GHz}$ relaxation oscillation frequency at $5.3 \mathrm{~mA}$, which roughly corresponds to the modulation frequency that separates the two situations in which the pulses switch off or do not switch off completely at each modulation cycle. In such a way, our experimental findings on the nonlinear dynamical behavior of a multitransverse-mode VCSEL when subject to high-frequency current modulation show that irregular pulsating dynamics can be obtained for a wide range of modulation frequencies as a result of the competition between the different transverse modes of the laser, in agreement with the theoretical predictions.

\section{Polarization Dynamics of VCSEL with Optical Feedback: Coherence Resonance in Optical Bistable Time-Delayed System}

Time-delayed optical feedback (OF) perturbs the steady state laser emission and leads to complex dynamics [40]. When a VCSEL is subject to isotropic (the same for any light polarization) $\mathrm{OF}$, the route to dynamical instabilities is modified-see Figure 10 [62]. Due to the small frequency difference between the two solitary VCSEL LP modes, the modulation of the threshold gain as a result of the $\mathrm{OF}$ for the ECMs of different polarization differs. As can be seen from Figure 10(a), the change of the VCSEL injection current periodically destabilizes the laser operation through a Hopf bifurcation to a limit cycle [62]. The amplitude of the periodic oscillation initially increases but then the VCSEL undergoes a PS to a stable emission in the orthogonal LP state. Actually, the injection current, via the current induced 


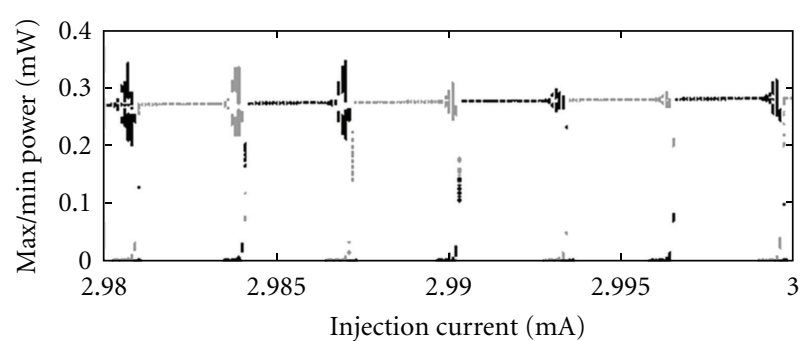

(a)

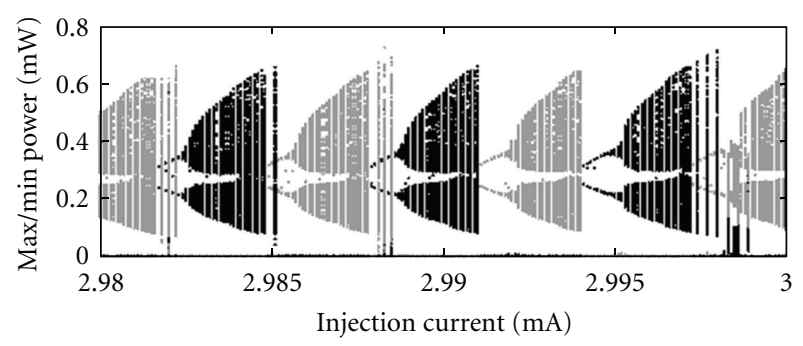

(b)

FIGURE 10: Numerically calculated bifurcation diagrams as a function of the injection current for external mirror reflectivity of $r_{\mathrm{ex}}=$ 0.005 (a) and $r_{\text {ex }}=0.02$ (b). The two LP modes are represented with solid black ( $x$-LP mode) and gray ( $y$-LP mode) lines.

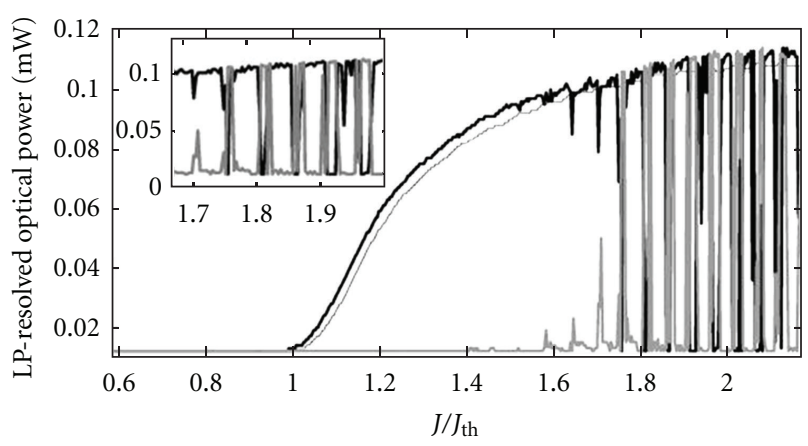

FIgUre 11: Experimental LI curve of a VCSEL subject to a weak optical feedback (thick lines), to be compared with the case without optical feedback (thin line). The $x$-LP $(y$-LP) mode intensity is plotted in black (gray). The inset shows enlargement to better illustrate the channeled LI curve with multiple PS points. After [61]. (Reprinted by permission from Opt. Lett.)

heating and the corresponding refractive index change, modifies the wavelength of the emitted light and therefore the phase of the light fed back into the VCSEL. In such a way, periodic regions of alternating lasing in the two LP modes are formed. For a fourfold increased reflectivity of the external mirror, the PS occurs between unstable LP modes, with different degree of destabilization-see Figure 10(b). Such a channeled light versus current (LI) curve has been found experimentally in $[61,63]$ and is shown in Figure 11 [61].

If we set the injection current close to a PS point light randomly alternates between emission of the $x$-LP mode and emission of the $y$-LP mode (see Figure 12). This situation

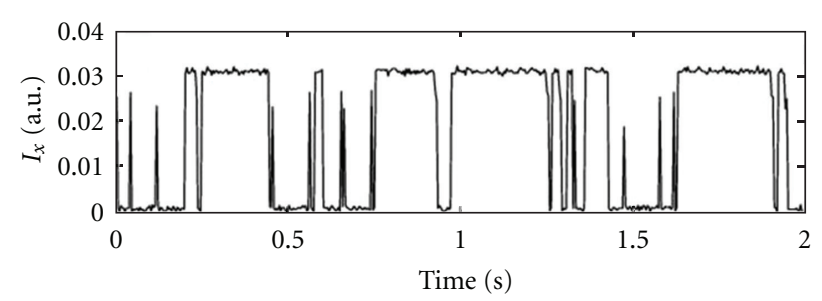

(a)

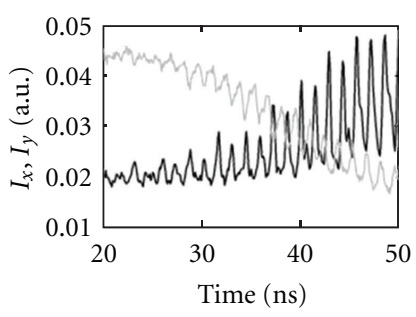

(b)

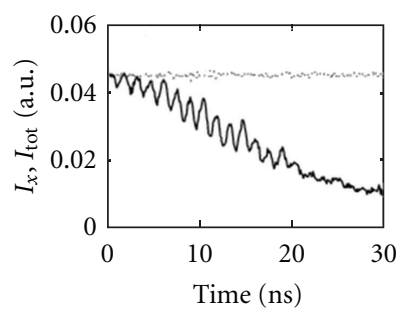

(c)
Figure 12: (a) Typical time-trace of mode hopping in the LP mode intensity of a VCSEL close to a polarization switching induced by weak optical feedback. (b) is an enlargement of (a) showing the two LP mode intensities when the light polarization hops between the two orthogonal directions. (c) shows a time-trace of the LP mode intensity (solid curve) together with the one of the total intensity (dotted curve). After [61]. (Reprinted by permission from Opt. Lett.)

is called polarization mode hopping for the case of solitary VCSEL [64-66], and from its statistical analysis it appears that the dwell time in a LP mode follows a Kramers law, that is, the probability distribution function exponentially decays with time. The mean dwell time depends on the injection current and the spontaneous emission level. In our OF experiment, superimposed on the slow polarization mode hopping (Figure 12(a)), a fast oscillatory behavior appears, at the frequency of the external cavity $(750 \mathrm{MHz})$, that is, the inverse of the OF time delay (Figure 12(b)). These fast oscillations more clearly appear during an attempt to obtain successful polarization switch. The two LP intensities are strongly anticorrelated and therefore the oscillations vanish in the time trace of the total intensity (Figure 12(c)). It has been theoretically, [67] and experimentally [68] demonstrated that Kramers law is violated for VCSELs with OF: the time-delay strongly influences the residence time distribution leading to the appearance of an oscillatory behavior with peaks at the delay time and a valley connecting the statistics for short- and long-dwell times [68].

The coexistence of stochastic (noise driven) process of polarization mode hoping with the deterministic oscillatory behavior at the delay time brings an interesting question about the possibility to observe a coherence resonance in such a system, that is, a resonance of the dynamical regularity of the system at a certain noise level. It is indeed commonly accepted now that noise can play constructive role in nonlinear dynamical systems. After the seminal paper of Benzi et al. [69], the phenomenon of stochastic resonance, namely, the fact that adding noise can better 


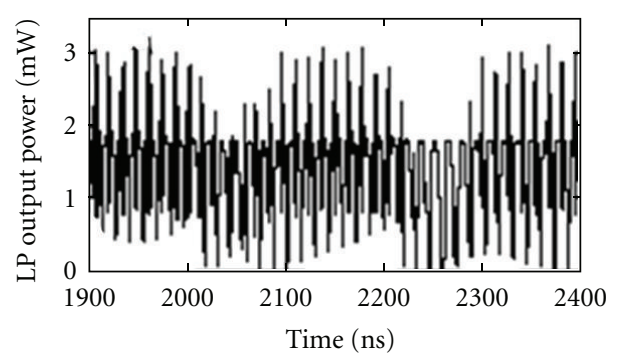

(a)

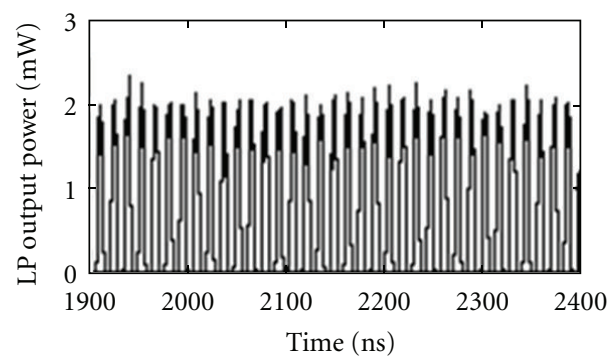

(b)

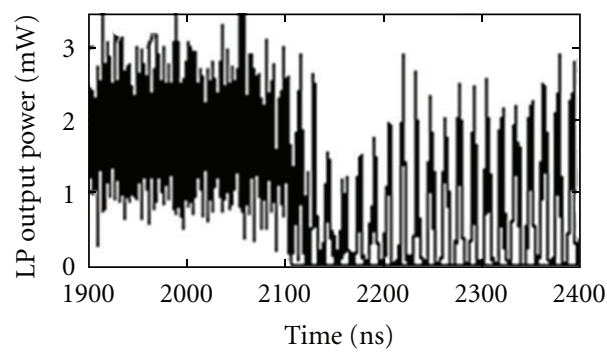

(c)

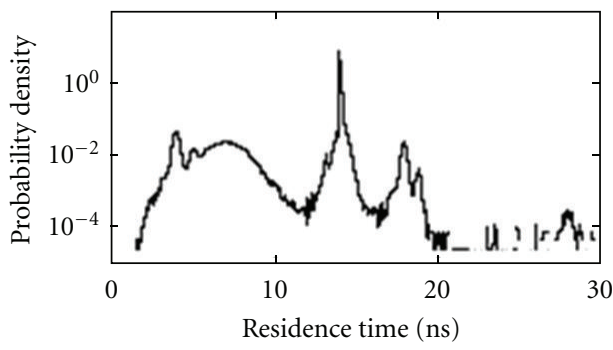

(d)

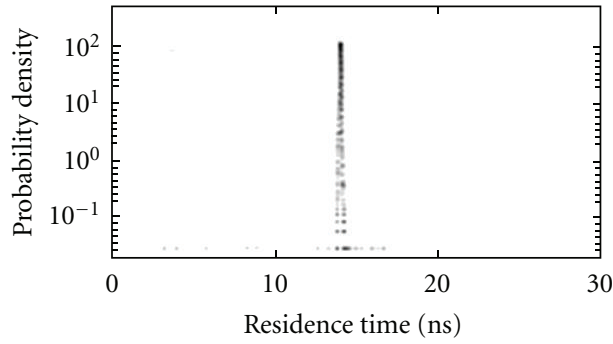

(e)

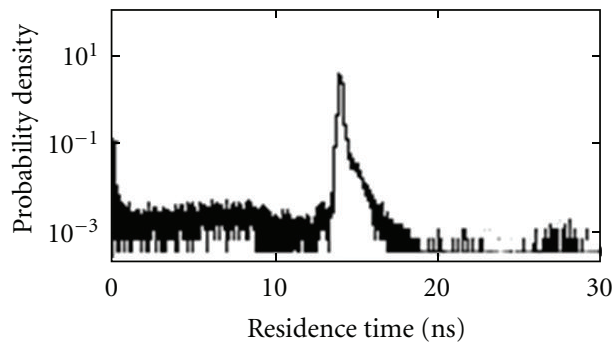

(f)

Figure 13: Numerical time traces of the $x$-LP mode (a), (b), (c), and joint residence-time distributions (d), (e), (f) for the case of long delay time $(\tau=13 \mathrm{~ns})$ and for different values of the external noise: (a), (d): $J_{\mathrm{var}}=1.0610 \_7$; (b), (e): $J_{\mathrm{var}}=0.0066$; (c), (f): $J_{\mathrm{var}}=0.0266$. After [68]. (Reprinted by permission from Phys. Rev. A.)

synchronize dynamical system to an external periodic signal, has attracted a lot of interest in biology [70], physics [71], chemistry [72], and so forth (for a review see e.g., [73]). It has recently been realized that noise can enhance regular dynamics in nonlinear systems even in absence of external signal, when an internal time scale is present in the system [74]. This phenomenon is initially considered as stochastic resonance in autonomous system and lately named coherence resonance (CR) [75]. CR has been first predicted for excitable dynamical systems [74-77], that is, systems that emit quasiregular pulses as a result of an excitation threshold and with a refractory period. It has been demonstrated experimentally in semiconductor lasers subject to optical feedback and driven into chaotic excitable dynamics [78]. For a recent review see [79]. Theoretical works on different models furthermore reported that not only excitable but also bistable or multistable systems driven by noise can exhibit CR [80, 81], as also confirmed experimentally in bistable chaotic electronic Chua circuits [82]. Recently, CR has been predicted in another class of systems, which exhibit bistability together with time-delay [83]. These systems are interesting considering also that delay is an inherent property of Nature, with examples found in biology [84] and economics [85].

A VCSEL subject to optical feedback is an example of a bistable time-delayed system and CR has been indeed observed in this system first numerically [68] and then experimentally [86]. The numerical prediction of CR for a VCSEL subject to isotropic OF is shown in Figure 13 [68]. In Figures 13(a)-13(c), we show the polarization resolved time traces of the output power for increased strength of the external noise added to the injection current and in Figures 13(d)-13(h) the corresponding joint residence time distribution (RTD). As can be seen from Figure 13, coherence resonance is indeed present in such a system. A continuous increase of the strength of the external noise results first in an increase of the peak amplitude and a decrease of the width of the joint RTD maximum at the delay time. The peak amplitude reaches a maximum and the peak width reaches a minimum for a given value of the noise (Figures 13(b), 13(e)), where the mode-hopping regime exhibits the most regular periodic behavior at the external cavity (EC) round-trip time. For larger external noise strengths, the RTD exhibits smaller peak amplitude at 


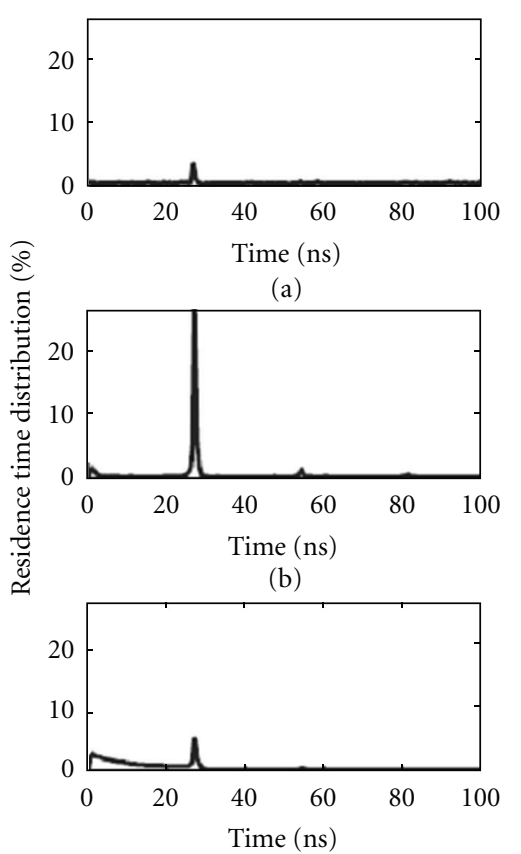

(c)
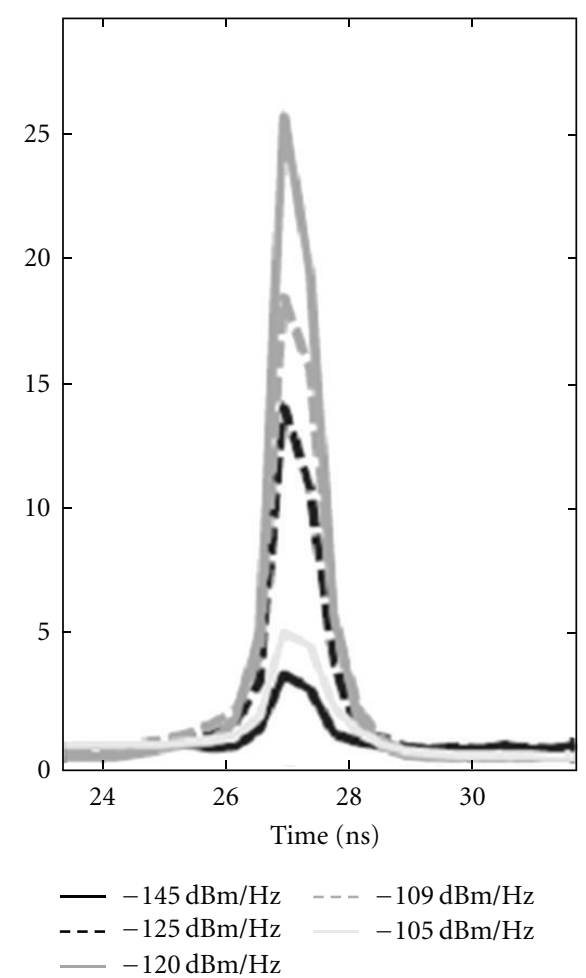

(d)

FIGURE 14: (a)-(c) Joint residence time distribution for three increasing noise levels, showing an optimal regularity at the delay time for an optimal noise level (case b). (d) is an enlargement of the JRTD around time values corresponding to the delay time, and for increasing values of noise added to the injection current. After [86]. (Reprinted by permission from Phys. Rev. Lett.)

the delay time and broadens towards smaller and larger time values. The mode-hopping time-trace exhibits windows of regular dynamics at the EC round-time but in general the dynamics is dominated by noise.

In order to observe CR experimentally, we have made use of the great possibility for tuning the PS current and hysteresis width by OF from an extremely short external cavity with a length of several $10 \mu \mathrm{m}[88,89]$. The VCSEL is subject to OF from two external cavities. The first one is formed between the VCSEL output mirror and a fiber facet with enhanced reflectivity of $23 \%$ at a close distance (of the order of $10 \mu \mathrm{m}$ ), that is, it is an extremely short external cavity used to tune the VCSEL polarizationswitching current. The second cavity is the optical fiber itself and introduces a time delay of $\tau=27 \mathrm{~ns}$ in the system. To look for a CR, an electrical noise with zero mean value and a flat spectrum until $300 \mathrm{MHz}$ is added to the injection current of the VCSEL. The effect of the noise level on the distribution of the joint residence time (JRTD) is shown in Figure 14. If the noise is weak, the system needs a lot of time to consecutively visit the two stable states and the peak of the JRTD at the external cavity round trip time ( $27 \mathrm{~ns})$ is very small (d). As the noise strength is increased, the peak at $27 \mathrm{~ns}$ dramatically increases reaching its maximum (e). For higher noise intensities, more and more fast PSs occur and the background masks the peak structure (f). The right panel (d) confirms the existence of an optimal noise level of $-120 \mathrm{dBm} / \mathrm{Hz}$ for which the JRTD exhibits a maximum peak at the delay time. This constitutes a clear evidence of coherence resonance in our system. Different indicators have complemented our observations. In particular we have observed the radio-frequency (RF) spectrum of the laser output for different noise levels. A peak appears close to the long external cavity frequency and the peak height relative to the RF spectrum noise floor reaches a maximum for a given noise level (similar to the noise level that brings the maximum JRTD value at the delay time).

\section{Light Polarization Specificities of Chaos Synchronization of Unidirectionally Coupled VCSEL}

The growing interest in implementing chaos communication using VCSELs motivates further investigations to better understand the influence of polarization dynamics on the synchronization properties. In this section, we present a theoretical investigation of the effect of polarization mode competition on the synchronization characteristics of two unidirectionally coupled VCSELs [87]. The master VCSEL only is rendered chaotic by optical feedback, and its chaotic output is coupled to the slave VCSEL by use of an isotropic optical injection. We show that, depending on the injection conditions, the synchronization quality can be strongly enhanced when the master laser and therefore also the synchronized slave exhibit two LP mode dynamics. 


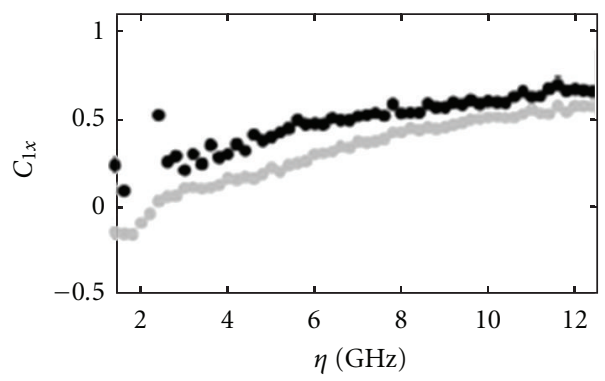

(a1)

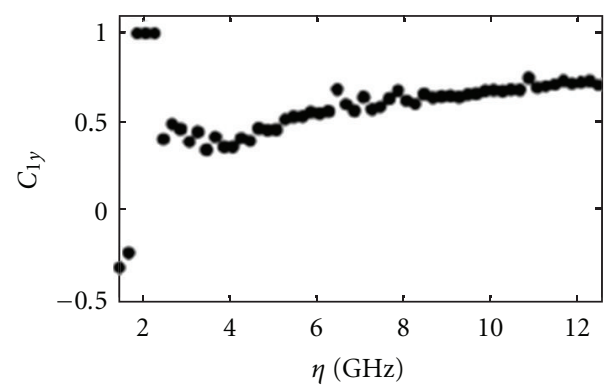

(b1)

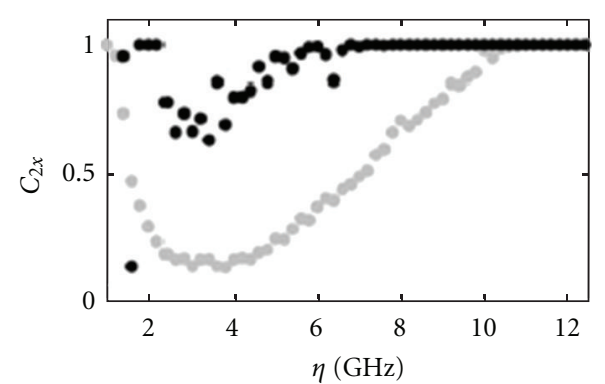

(a2)

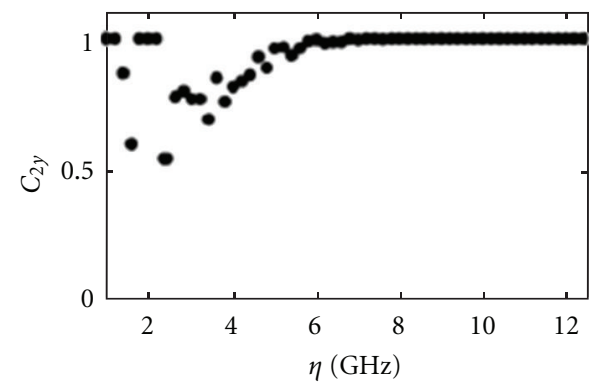

(b2)

FIGURE 15: Evolution of the correlation coefficients related to isochronous (a1) and (b1) and anticipative (a2) and (b2) synchronization as a function of $\eta$. The gray (black) color corresponds to the case of single-mode (two-mode) chaos. After [87]. (Reprinted by permission from Opt. Lett.)

In isotropic optical feedback/injection configuration, the two VCSELs can exhibit either an anticipating or isochronous (injection-locking) type of synchronization [90]. Anticipating (perfect) synchronization is achieved when laser intensities for the $x$ - and $y$-polarizations of the master laser as well as its carrier density at time $t$ are synchronized with the corresponding light intensities and carrier density of the slave laser at time $t-\tau$, that is, $I_{x, y}^{m}(t)=a I_{x, y}^{s}(t-\tau)$. Necessary conditions for the existence of such a solution are that both lasers exhibit the same device parameters, bias currents, zero detuning, and also $f=\eta$ with $f$ being the feedback strength and $\eta$ the coupling (injection) strength. The isochronous synchronization solution is of the type $I_{x, y}^{m}(t)=a I_{x, y}^{s}(t)$, with $a$ being a constant. To evaluate the synchronization quality and to discriminate between isochronous and anticipative synchronizations, we use the cross-correlation coefficients for the $x$ - and $y$-LP mode intensities $C_{1 x, y}(t, t)$ and $C_{2 x, y}(t+$ $\tau, t)$, respectively.

Figure 15 shows the evolution of the isochronous and the anticipative correlation coefficients when the injection rate $\eta$ is varied but still equal to the feedback rate $f$. In Figures 15(a1) and 15(a2), we add gray curves that show the evolution of $C_{1 x}$ and $C_{2 x}$ in the case of single $x$-LP mode chaotic dynamics. In the whole range of $\eta, C_{2}>$ $C_{1}$, whatever the LP modes that are analyzed ( $x$ or $y$ ) and independently of the single $x$-LP mode or two LP mode cases in the laser dynamics (black or gray curves). The lasers therefore exhibit anticipative synchronization. The still large isochronous correlation coefficient $C_{1}$ can be related to the presence of a periodicity at $\tau$ in the intensity time traces. For $\eta<3 \mathrm{~ns}^{-1}$ the lasers exhibit either weakly synchronized irregular dynamics or locked steady states (see the small range of $\eta$ with perfect synchronization in the two LP mode case). For larger $\eta$, the synchronization quality improves with $\eta$. Indeed, the lasers exhibit desynchronization bursts, but the average time between them increases as $\eta$ increases, leading to better synchronization over a given time window. The comparison between the black and gray curves in Figures 15(a1) and 15(a2) unveils, moreover, that the correlation coefficients (and therefore the synchronization quality) improve in the two LP mode cases. Therefore, the synchronization quality between unidirectionally coupled VCSELs can be significantly enhanced when the feedbackinduced chaos in the master laser involves both orthogonal LP fundamental transverse modes. Two regions of enhanced synchronization between the injected LP mode and the corresponding slave LP mode are identified in [91]. In the so-called region II, the slave VCSEL exhibits anticorrelated dynamics in its two LP modes while in the so-called region I, the slave VCSEL exhibits dynamics in only one LP mode, which corresponds to the polarization of the injected field. The two regions exhibit different synchronization properties in both the LP mode dynamics and total intensity dynamics.

\section{Conclusions}

In conclusions, we have shown that the nonlinear dynamics of vertical-cavity surface-emitting lasers (VCSELs) induced by optical injection, current modulation optical feedback 
and unidirectional coupling are drastically modified due to the underlying polarization and transverse mode competition. This provides new features to the rich nonlinear dynamics such as, for the case of optical injection, the new Hopf bifurcation on a two-polarization-mode solution that delimits the injection locking region and the new resonance tongue of polarization switching and injection locking of first-order transverse mode for large positive detunings. Similarly, the underlying polarization mode competition leads to chaotic-like behavior in case of gain switching and the presence of two transverse modes additionally reduces the possibility of regular dynamics.

Since the polarization selection is very weak, a small amount of optical feedback is enough to significantly modify the VCSEL polarization behavior and observe laser dynamics that significantly differ from those typically observed in edge-emitting lasers. Typical dynamical instabilities such as low-frequency fluctuations and self-pulsations induced by optical feedback still exist in VCSELs but with the additional complexity of the anticorrelated dynamics between polarization modes. The bistable property of VCSEL makes it possible to investigate very fundamental problems of bistable systems with time-delay, such as the coherence resonance phenomenon. We also demonstrated that the synchronization quality between unidirectionally coupled VCSELs can be significantly enhanced when the feedback-induced chaos in the master laser involves both orthogonal LP fundamental transverse modes.

\section{Acknowledgments}

Some of the works presented in this paper have been undertaken with other past and present colleagues and coworkers from other groups, as acknowledged via the relevant journal paper citations. The authors also acknowledge the support of COST MP0702 European Action, Conseil Régional de Lorraine, Fondation Supélec, IAP 6/10-Belgian Science Policy, FWO Flanders, GOA, and OZR of the VUB.

\section{References}

[1] H. Li and K. Iga, Eds., Vertical-Cavity Surface-Emitting Laser Devices, vol. 6 of Springer Series in Photonics, Springer, Berlin, Germany, 2002.

[2] C. J. Chang-Hasnain, J. P. Harbison, G. Hasnain, A. C. Von Lehmen, L. T. Florez, and N. G. Stoffel, "Dynamic, polarization, and transverse mode characteristics of vertical cavity surface emitting lasers," IEEE Journal of Quantum Electronics, vol. 27, no. 6, pp. 1402-1409, 1991.

[3] D. Vakhshoori, J. D. Wynn, G. J. Zydzik et al., “Top-surface emitting lasers with $1.9 \mathrm{v}$ threshold voltage and the effect of spatial hole burning on their transverse mode operation and efficiencies," Applied Physics Letters, vol. 62, no. 13, pp. 1448 1450, 1993.

[4] A. Valle, J. Sarma, and K. A. Shore, "Spatial holeburning effects on the dynamics of vertical cavity surface-emitting laser diodes," IEEE Journal of Quantum Electronics, vol. 31, no. 8, pp. 1423-1431, 1995.
[5] K. D. Choquette, R. P. Schneider, K. L. Lear, and R. E. Leibenguth, "Gain-dependent polarization properties of vertical-cavity lasers," IEEE Journal on Selected Topics in Quantum Electronics, vol. 1, no. 2, pp. 661-666, 1995.

[6] M. San Miguel, Q. Feng, and J. V. Moloney, "Lightpolarization dynamics in surface-emitting semiconductor lasers," Physical Review A, vol. 52, no. 2, pp. 1728-1739, 1995.

[7] J. Martin-Regalado, F. Prati, M. San Miguel, and N. B. Abraham, "Polarization properties of vertical-cavity surfaceemitting lasers," IEEE Journal of Quantum Electronics, vol. 33, no. 5, pp. 765-783, 1997.

[8] K. Panajotov, B. Ryvkin, J. Danckaert, M. Peeters, H. Thienpont, and I. Veretennicoff, "Polarization switching in VCSEL's due to thermal lensing," IEEE Photonics Technology Letters, vol. 10, no. 1, pp. 6-8, 1998.

[9] A. Valle, K. A. Shore, and L. Pesquera, "Polarization selection in birefringent vertical-cavity surface emitting lasers," Journal of Lightwave Technology, vol. 14, no. 9, pp. 2062-2068, 1996.

[10] B. Ryvkin, K. Panajotov, A. Georgievski et al., "Effect of photon-energy-dependent loss and gain mechanisms on polarization switching in vertical-cavity surface-emitting lasers," Journal of the Optical Society of America B, vol. 16, no. 11, pp. 2106-2113, 1999.

[11] J. I. Nishizawa and K. Ishida, "Injection-induced modulation of laser light by the interaction of laser diodes," IEEE Journal of Quantum Electronics, vol. 11, no. 7, pp. 515-519, 1975.

[12] R. Lang, "Injection locking properties of a semiconductor laser," IEEE Journal of Quantum Electronics, vol. 18, no. 6, pp. 976-983, 1982.

[13] K. Iwashita and K. Nakagawa, "Suppression of mode partition by laser diode light injection," IEEE Journal of Quantum Electronics, vol. 18, no. 10, pp. 1669-1674, 1982.

[14] T. B. Simpson, J. M. Liu, and A. Gavrielides, "Bandwidth enhancement and broadband noise reduction in injectionlocked semiconductor lasers," IEEE Photonics Technology Letters, vol. 7, no. 7, pp. 709-711, 1995.

[15] L. Goldberg, H. F. Taylor, J. F. Weller, and D. R. Scifres, "Injection locking of coupled-stripe diode laser arrays," Applied Physics Letters, vol. 46, no. 3, pp. 236-238, 1985.

[16] E. K. Lee, H. S. Pang, J. D. Park, and H. Lee, "Bistability and chaos in an injection-locked semiconductor laser," Physical Review A, vol. 47, no. 1, pp. 736-739, 1993.

[17] V. Annovazzi-Lodi, S. Donati, and M. Manna, "Chaos and locking in a semiconductor laser due to external injection," IEEE Journal of Quantum Electronics, vol. 30, no. 7, pp. 15371541, 1994.

[18] T. B. Simpson, J. M. Liu, A. Gavrielides, V. Kovanis, and P. M. Alsing, "Period-doubling cascades and chaos in a semiconductor laser with optical injection," Physical Review A, vol. 51, no. 5, pp. 4181-4185, 1995.

[19] P. M. Varangis, A. Gavrielides, T. Erneux, V. Kovanis, and L. F. Lester, "Frequency entrainment in optically injected semiconductor lasers," Physical Review Letters, vol. 78, no. 12, pp. 2353-2356, 1997.

[20] S. Wieczorek, B. Krauskopf, and D. Lenstra, "Multipulse excitability in a semiconductor laser with optical injection," Physical Review Letters, vol. 88, no. 6, pp. 063901/1-063901/4, 2002.

[21] Z. G. Pan, S. Jiang, M. Dagenais et al., "Optical injection induced polarization bistability in vertical-cavity surfaceemitting lasers," Applied Physics Letters, vol. 63, no. 22, pp. 2999-3001, 1993.

[22] J. B. Altés, I. Gatare, K. Panajotov, H. Thienpont, and M. Sciamanna, "Mapping of the dynamics induced by orthogonal 
optical injection in vertical-cavity surface-emitting lasers," IEEE Journal of Quantum Electronics, vol. 42, no. 2, pp. 198207, 2006.

[23] Y. Hong, P. S. Spencer, P. Rees, and K. Alan Shore, "Optical injection dynamics of two-mode vertical cavity surfaceemitting semiconductor lasers," IEEE Journal of Quantum Electronics, vol. 38, no. 3, pp. 274-278, 2002.

[24] H. Li, T. L. Lucas, J. G. McInerney, M. W. Wright, and R. A. Morgan, "Injection locking dynamics of vertical cavity semiconductor lasers under conventional and phase conjugate injection," IEEE Journal of Quantum Electronics, vol. 32, no. 2, pp. 227-235, 1996.

[25] J. Y. Law, G. H. M. Van Tartwijk, and G. P. Agrawal, "Effects of transverse-mode competition on the injection dynamics of vertical-cavity surface-emitting lasers," Journal of Optics B, vol. 9, no. 5, pp. 737-747, 1997.

[26] S. Bandyopadhyay, Y. Hong, P. S. Spencer, and K. A. Shore, "Experimental observation of anti-phase polarisation dynamics in VCSELS," Optics Communications, vol. 202, no. 1-3, pp. 145-154, 2002.

[27] Y. Hong, P. Spencer, S. Bandyopadhyay, P. Rees, and K. A. Shore, "Polarization resolved chaos and instabilities in a VCSEL subject to optical injection," Optics Communications, vol. 216, pp. 185-187, 2003.

[28] C. H. Lee, T. H. Yoon, and S. Y. Shin, "Period doubling and chaos in a directly modulated laser diode," Applied Physics Letters, vol. 46, no. 1, pp. 95-97, 1985.

[29] Y. C. Chen, H. G. Winful, and J. M. Liu, "Subharmonic bifurcations and irregular pulsing behavior of modulated semiconductor lasers," Applied Physics Letters, vol. 47, no. 3, pp. 208 210, 1985.

[30] H. F. Liu and W. F. Ngai, "Nonlinear dynamics of a directly modulated $1.55 \mu \mathrm{m}$ InGaAsP distributed feedback semiconductor laser," IEEE Journal of Quantum Electronics, vol. 29, no. 6, pp. 1668-1675, 1993.

[31] Y. Matsui, S. Kutsuzawa, S. Arahira, Y. Ogawa, and A. Suzuki, "Bifurcation in 20-GHz gain-switched $1.55-\mu \mathrm{M}$ MQW lasers and its control by CW injection seeding," IEEE Journal of Quantum Electronics, vol. 34, no. 7, pp. 1213-1222, 1998.

[32] C. Mayol, R. Toral, C. R. Mirasso, S. I. Turovets, and L. Pesquera, "Theory of main resonances in directly modulated diode lasers," IEEE Journal of Quantum Electronics, vol. 38, no. 3, pp. 260-269, 2002.

[33] S. F. Yu, "Nonlinear dynamics of vertical-cavity surfaceemitting lasers," IEEE Journal of Quantum Electronics, vol. 35, no. 3, pp. 332-340, 1999.

[34] A. Valle, L. Pesquera, S. I. Turovets, and J. M. López, "Nonlinear dynamics of current-modulated vertical-cavity surfaceemitting lasers," Optics Communications, vol. 208, no. 1-3, pp. 173-182, 2002.

[35] J. Y. Law and G. P. Agrawal, "Nonlinear spatio-temporal dynamics due to transverse-mode competition in gain-switched microcavity semiconductor lasers," Optics Communications, vol. 138, no. 1-3, pp. 95-98, 1997.

[36] M. Sciamanna, A. Valle, P. Mégret, M. Blondel, and K. Panajotov, "Nonlinear polarization dynamics in directly modulated vertical-cavity surface-emitting lasers," Physical Review E, vol. 68, no. 1, Article ID 016207, 4 pages, 2003.

[37] A. Valle, M. Sciamanna, and K. Panajotov, "Irregular pulsating polarization dynamics in gain-switched vertical-cavity surface-emitting lasers," IEEE Journal of Quantum Electronics, vol. 44, no. 2, pp. 136-143, 2008.

[38] A. Valle, M. Sciamanna, and K. Panajotov, "Nonlinear dynamics of the polarization of multitransverse mode vertical-cavity surface-emitting lasers under current modulation," Physical Review E, vol. 76, no. 4, Article ID 046206, 2007.

[39] A. Valle, M. Arizaleta, H. Thienpont, K. Panajotov, and M. Sciamanna, "Transverse mode competition effects on the dynamics of gain-switched vertical-cavity surface-emitting lasers," Applied Physics Letters, vol. 93, no. 13, Article ID 131103, 2008.

[40] J. Mork, B. Tromborg, and J. Mark, "Chaos in semiconductor lasers with optical feedback: theory and experiment," IEEE Journal of Quantum Electronics, vol. 28, no. 1, pp. 93-108, 1992.

[41] C. Risch and C. Voumard, "Self-pulsation in the output intensity and spectrum of GaAs-AlGaAs cw diode lasers coupled to a frequency-selective external optical cavity," Journal of Applied Physics, vol. 48, no. 5, pp. 2083-2085, 1977.

[42] D. Lenstra, B. H. Verbeek, and A. J. den Boef, "Coherence collapse in single-mode semiconductor lasers due to optical feedback," IEEE Journal of Quantum Electronics, vol. 21, no. 6, pp. 674-679, 1984.

[43] R. W. Tkach and A. R. Chraplyvy, "Regimes of feedback effects in 1.5- $\mu \mathrm{m}$ distributed feedback lasers," Journal of Lightwave Technology, vol. 4, no. 11, pp. 1655-1661, 1986.

[44] C. Masoller and N. B. Abraham, "Low-frequency fluctuations in vertical-cavity surface-emitting semiconductor lasers with optical feedback," Physical Review A, vol. 59, no. 4, pp. 30213031, 1999.

[45] M. Sciamianna, C. Masoller, F. Rogister, P. Mégret, N. B. Abraham, and M. Blondel, "Fast pulsing dynamics of a vertical-cavity surface-emitting laser operating in the lowfrequency fluctuation regime," Physical Review A, vol. 68, no. 1, Article ID 015805, 4 pages, 2003.

[46] M. Giudici, S. Balle, T. Ackemann, S. Barland, and J. R. Tredicce, "Polarization dynamics in vertical-cavity surfaceemitting lasers with optical feedback: experiment and model," Journal of the Optical Society of America B, vol. 16, no. 11, pp. 2114-2123, 1999.

[47] M. Sondermann, H. Bohnet, and T. Ackermann, "Lowfrequency fluctuations and polarization dynamics in verticalcavity surface-emitting lasers with isotropic feedback," Physical Review A, vol. 67, no. 2, Article ID 021802(R), 4 pages, 2003.

[48] J. Dellunde, A. Valle, L. Pesquera, and K. A. Shore, "Transverse-mode selection and noise properties of external-cavity vertical-cavity surface-emitting lasers including multiplereflection effects," Journal of the Optical Society of America B, vol. 16, no. 11, pp. 2131-2139, 1999.

[49] F. Marino, S. Barland, and S. Balle, "Single-mode operation and transverse-mode control in VCSELs induced by frequency-selective feedback," IEEE Photonics Technology Letters, vol. 15, no. 6, pp. 789-791, 2003.

[50] L. M. Pecora and T. L. Carroll, "Synchronization in chaotic systems," Physical Review Letters, vol. 64, no. 8, pp. 821-824, 1990.

[51] C. R. Mirasso, P. Colet, and P. García-Fernández, "Synchronization of chaotic semiconductor lasers: application to encoded communications," IEEE Photonics Technology Letters, vol. 8, no. 2, pp. 299-301, 1996.

[52] R. Ju, P. S. Spencer, and K. A. Shore, "Polarization-preserved and polarization-rotated synchronization of chaotic verticalcavity surface-emitting lasers," IEEE Journal of Quantum Electronics, vol. 41, no. 12, pp. 1461-1467, 2005. 
[53] M. S. Torre, C. Masoller, and K. A. Shore, "Synchronization of unidirectionally coupled multi-transverse-mode verticalcavity surface-emitting lasers," Journal of the Optical Society of America B, vol. 21, no. 10, pp. 1772-1780, 2004.

[54] Y. Hong, M. W. Lee, P. S. Spencer, and K. A. Shore, "Synchronization of chaos in unidirectionally coupled vertical-cavity surface-emitting semiconductor lasers," Optics Letters, vol. 29, no. 11, pp. 1215-1217, 2004.

[55] I. Gatare, M. Sciamanna, J. Buesa, H. Thienpont, and K. Panajotov, "Nonlinear dynamics accompanying polarization switching in vertical-cavity surface-emitting lasers with orthogonal optical injection," Applied Physics Letters, vol. 88, no. 10, Article ID 101106, 2006.

[56] A. Valle, I. Gatare, K. Panajotov, and M. Sciamanna, "Transverse mode switching and locking in vertical-cavity surfaceemitting lasers subject to orthogonal optical injection," IEEE Journal of Quantum Electronics, vol. 43, no. 4, pp. 322-333, 2007.

[57] I. Gatare, M. Sciamanna, M. Nizette, and K. Panajotov, "Bifurcation to polarization switching and locking in verticalcavity surface-emitting lasers with optical injection," Physical Review A, vol. 76, no. 3, Article ID 031803, 2007.

[58] M. Sciamanna and K. Panajotov, "Two-mode injection locking in vertical-cavity surface-emitting lasers," Optics Letters, vol. 30, no. 21, pp. 2903-2905, 2005.

[59] M. Sciamanna and K. Panajotov, "Route to polarization switching induced by optical injection in vertical-cavity surface-emitting lasers," Physical Review A, vol. 73, no. 2, Article ID 023811, 17 pages, 2006.

[60] S. Wieczorek, B. Krauskopf, T. B. Simpson, and D. Lenstra, "The dynamical complexity of optically injected semiconductor lasers," Physics Reports, vol. 416, no. 1-2, pp. 1-128, 2005.

[61] M. Sciamanna, K. Panajotov, H. Thienpont, I. Veretennicoff, P. Mégret, and M. Blondel, "Optical feedback induces polarization mode hopping in vertical-cavity surface-emitting lasers," Optics Letters, vol. 28, no. 17, pp. 1543-1545, 2003.

[62] K. Panajotov, M. Arizaleta, V. Gomez et al., "Semiconductor lasers for quantum sensing," in Quantum Sensing and Nanophotonic Devices, Proceedings of SPIE, pp. 360-375, January 2004.

[63] P. Besnard, M. Chares, G. Stephan, and F. Robert, "Switching between polarization modes of a vertical-cavity surfaceemitting laser by isotropic optical feedback," Journal of the Optical Society of America B, vol. 16, pp. 1059-1063, 1999.

[64] G. Giacomelli, F. Marin, and I. Rabbiosi, "Stochastic and bona fide resonance: an experimental investigation," Physical Review Letters, vol. 82, no. 4, pp. 675-678, 1999.

[65] M. B. Willemsen, M. U. F. Khalid, M. P. Van Exter, and J. P. Woerdman, "Polarization switching of a vertical-cavity semiconductor laser as a Kramers hopping problem," Physical Review Letters, vol. 82, no. 24, pp. 4815-4818, 1999.

[66] B. Nagler, M. Peeters, J. Albert et al., "Polarization-mode hopping in single-mode vertical-cavity surface-emitting lasers: theory and experiment," Physical Review A, vol. 68, no. 1, Article ID 013813, 8 pages, 2003.

[67] C. Masoller, "Distribution of residence times of time-delayed bistable systems driven by noise," Physical Review Letters, vol. 90, no. 2, Article ID 020601, 4 pages, 2003.

[68] K. Panajotov, M. Sciamanna, A. Tabaka et al., "Residence time distribution and coherence resonance of optical-feedbackinduced polarization mode hopping in vertical-cavity surfaceemitting lasers," Physical Review A, vol. 69, no. 1, Article ID 011801(R), 4 pages, 2004.
[69] R. Benzi, A. Sutera, and A. Vulpiani, "The mechanism of stochastic resonance," Journal of Physics A, vol. 14, no. 11, pp. L453-L457, 1981.

[70] J. K. Douglass, L. Wilkens, E. Pantazelou, and F. Moss, "Noise enhancement of information transfer in crayfish mechanoreceptors by stochastic resonance," Nature, vol. 365, no. 6444, pp. 337-340, 1993.

[71] B. McNamara, K. Wiesenfeld, and R. Roy, "Observation of stochastic resonance in a ring laser," Physical Review Letters, vol. 60 , no. 25 , pp. 2626-2629, 1988.

[72] W. Hohmann, J. Müller, and F. W. Schneider, "Stochastic resonance in chemistry. 3. The minimal-bromate reaction," Journal of Physical Chemistry, vol. 100, no. 13, pp. 5388-5392, 1996.

[73] L. Gammaitoni, P. Hänggi, P. Jung, and F. Marchesoni, "Stochastic resonance," Reviews of Modern Physics, vol. 70, no. 1, pp. 223-287, 1998.

[74] H. Gang, T. Ditzinger, C. Z. Ning, and H. Haken, "Stochastic resonance without external periodic force," Physical Review Letters, vol. 71, no. 6, pp. 807-810, 1993.

[75] A. S. Pikovsky and J. Kurths, "Coherence resonance in a noisedriven excitable system," Physical Review Letters, vol. 78, no. 5, pp. 775-778, 1997.

[76] A. Neiman, P. I. Saparin, and L. Stone, "Coherence resonance at noisy precursors of bifurcations in nonlinear dynamical systems," Physical Review E, vol. 56, no. 1, pp. 270-273, 1997.

[77] S. Fauve and F. Heslot, "Stochastic resonance in a bistable system," Physics Letters A, vol. 97, no. 1-2, pp. 5-7, 1983.

[78] G. Giacomelli, M. Giudici, S. Balle, and J. R. Tredicce, "Experimental evidence of coherence resonance in an optical system," Physical Review Letters, vol. 84, no. 15, pp. 3298-3301, 2000.

[79] B. Lindner, J. García-Ojalvo, A. Neiman, and L. SchimanskyGeier, "Effects of noise in excitable systems," Physics Reports, vol. 392, no. 6, pp. 321-424, 2004.

[80] B. Lindner and L. Schimansky-Geier, "Coherence and stochastic resonance in a two-state system," Physical Review E, vol. 61, no. 6 B, pp. 6103-6110, 2000.

[81] C. Masoller, "Noise-induced resonance in delayed feedback systems," Physical Review Letters, vol. 88, no. 3, Article ID 034102, 4 pages, 2002.

[82] C. Palenzuela, R. Toral, C. R. Mirasso, O. Calvo, and J. D. Gunton, "Coherence resonance in chaotic systems," Europhysics Letters, vol. 56, no. 3, pp. 347-353, 2001.

[83] L. S. Tsimring and A. Pikovsky, "Noise-induced dynamics in bistable systems with delay," Physical Review Letters, vol. 87, no. 25, Article ID 250602, 4 pages, 2001.

[84] L. Nunney, "The effect of long time delays in predator-prey systems," Theoretical Population Biology, vol. 27, no. 2, pp. 202-221, 1985.

[85] M. C. Mackey, "Commodity price fluctuations: price dependent delays and nonlinearities as explanatory factors," Journal of Economic Theory, vol. 48, no. 2, pp. 497-509, 1989.

[86] M. Arizaleta Arteaga, M. Valencia, M. Sciamanna, H. Thienpont, M. López-Amo, and K. Panajotov, "Experimental evidence of coherence resonance in a time-delayed bistable system," Physical Review Letters, vol. 99, no. 2, Article ID 023903, 4 pages, 2007.

[87] I. Gatare, M. Sciamanna, A. Locquet, and K. Panajotov, "Influence of polarization mode competition on the synchronization of two unidirectionally coupled vertical-cavity surface-emitting lasers," Optics Letters, vol. 32, no. 12, pp. 1629-1631, 2007.

[88] M. A. Arteaga, H. J. Unold, J. M. Ostermann, R. Michalzik, H. Theinpont, and K. Panajotov, "Investigation of polarization 
properties of VCSELs subject to optical feedback from an extremely short external cavity-part I: theoretical analysis," IEEE Journal of Quantum Electronics, vol. 42, no. 2, pp. 89$101,2006$.

[89] M. A. Arteaga, M. López-Amo, H. Thienpont, and K. Panajotov, "Role of external cavity reflectivity for achieving polarization control and stabilization of vertical cavity surface emitting laser," Applied Physics Letters, vol. 90, no. 3, Article ID 031117, 3 pages, 2007.

[90] A. Locquet, F. Rogister, M. Sciamanna, P. Mégret, and M. Blondel, "Two types of synchronization in unidirectionally coupled chaotic external-cavity semiconductor lasers," Physical Review E, vol. 64, no. 4, Article ID 045203(R), 4 pages, 2001.

[91] M. Sciamanna, I. Gatare, A. Locquet, and K. Panajotov, "Polarization synchronization in unidirectionally coupled verticalcavity surface-emitting lasers with orthogonal optical injection," Physical Review E, vol. 75, no. 5, Article ID 056213, 10 pages, 2007. 

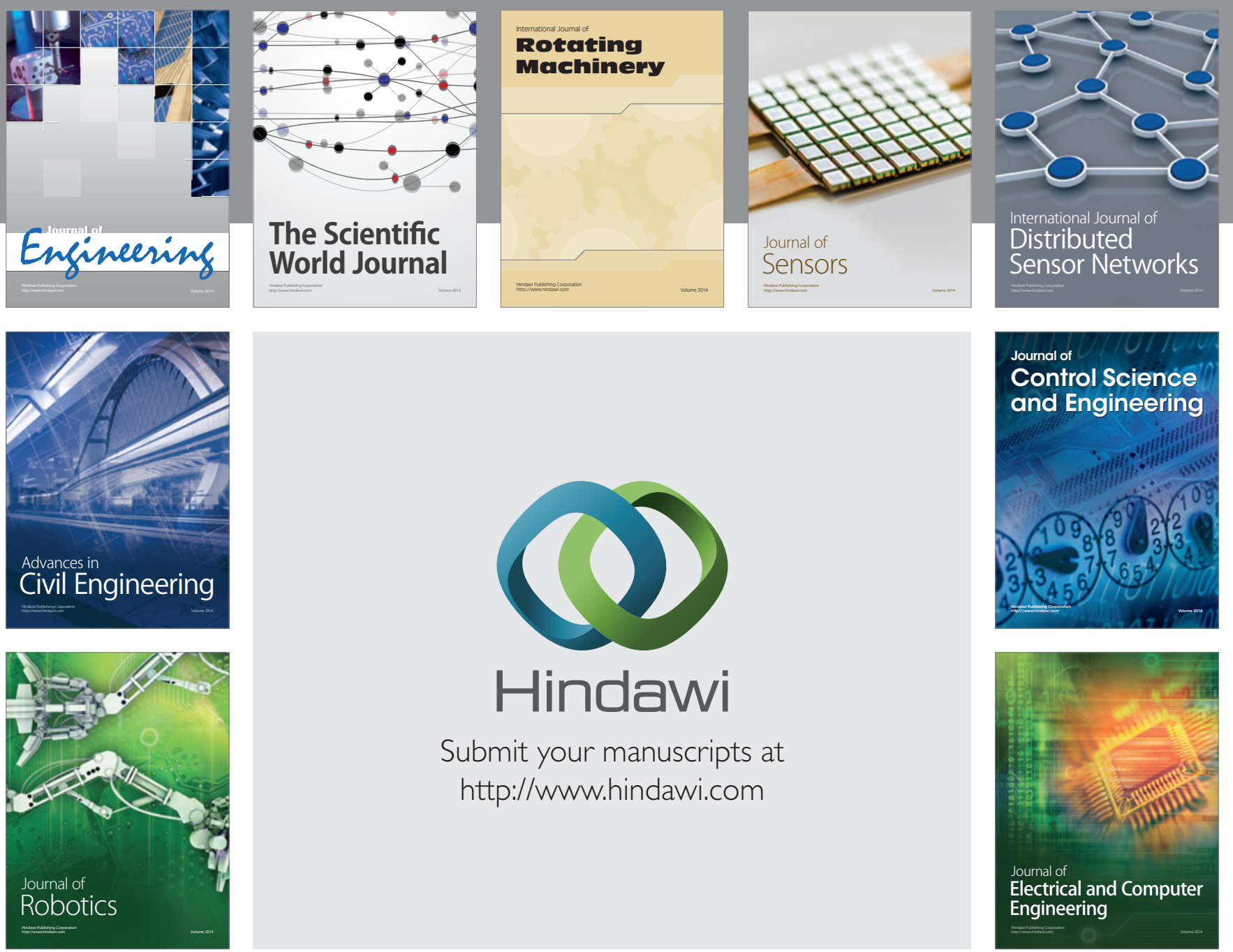

Submit your manuscripts at

http://www.hindawi.com
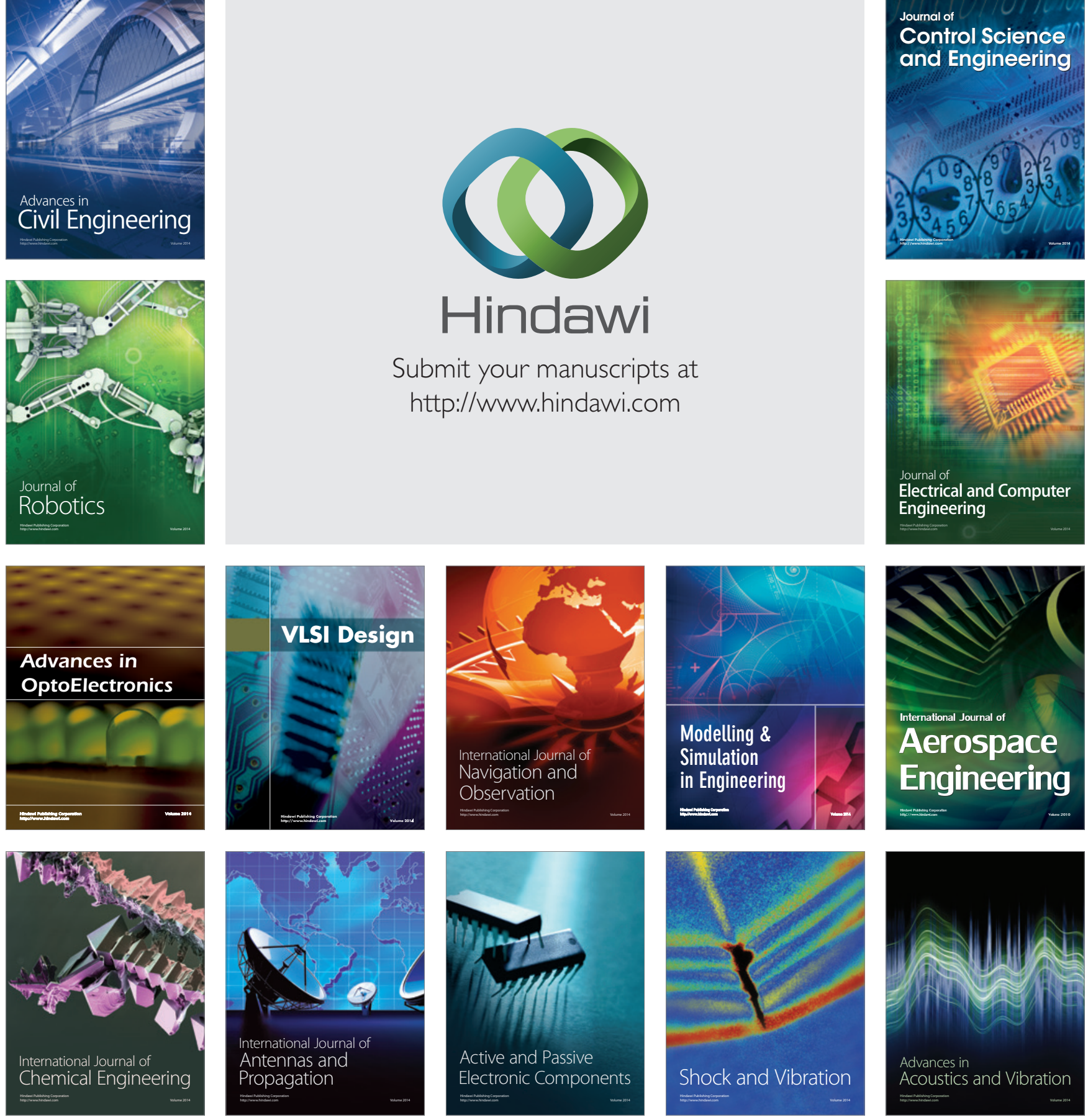